\title{
Valuation of Annuity Guarantees Under a Self-Exciting Switching Jump Model
}

\author{
Charles Guy Njike Leunga $^{1}$ (D) . Donatien Hainaut ${ }^{1}$
}

Received: 27 February 2021 / Revised: 10 January 2022 / Accepted: 18 January 2022 /

Published online: 9 February 2022

(c) The Author(s), under exclusive licence to Springer Science+Business Media, LLC, part of Springer Nature 2022

\begin{abstract}
This article investigates the valuation of annuity guarantees under a regime-switching model when the dynamics of the underlying stock price follow a self-exciting switching jump-diffusion process. In this framework, we add a jump component to a regime-switching geometric Brownian for large shocks on the stock price. The intensity of shock arrivals is a Hawkes process modulated by a continuous time hidden Markov chain with a finite number of states. The interest rate used for discounting is stochastic and correlated to the stock market. In an incomplete market, we define an equivalent martingale measure to price a variable annuity contract that guarantees a minimum living or death benefit. Under this equivalent martingale measure, we propose closed-form approximation formulas using the inverse Fourier transform technique. A numerical implementation highlights the impact of self-exciting jumps and economic regimes on the valuation of guarantees.
\end{abstract}

Keywords Variable annuity $\cdot$ Self-exciting $\cdot$ Hidden markov chain $\cdot$ Fourier transform

\section{Introduction}

Over the past decade, the design of life insurance products has been progressively adapted to customer needs, from classic pure endowments, death deferred capital or unit-linked insurance products to variable annuities (VAs). A variable annuity is a unit-linked annuity product with guarantees or riders, which are mainly living and death benefits. Such a product allows for the allocation of savings to either a pre- or post-retirement investment strategy, as it guarantees lifetime income or death capital and protects VA holders from

Charles Guy Njike Leunga

charles.njikeleunga@uclouvain.be

Donatien Hainaut

donatien.hainaut@uclouvain.be

1 Institute of Statistics, Biostatistics and Actuarial science, Université catholique de Louvain, Ottignies-Louvain-la-Neuve, Belgium 
market downturns. In addition to these advantages, the popularity of VAs stems from the fact that they are indirect tax-deferred investments. Unlike in the United States, where sales volumes of VAs amounts to more than $\$ 20$ billion per quarter ${ }^{1}$, in Europe the VA market is still expanding according to the annual reports of major European VA issuers. However, due to rider features of VAs, any VA issuer faces various risks, including financial, mortality or longevity and surrender risk. Furthermore, the low or negative interest rates environment, the interconnection of the markets and the interplay between risks are features that make the fair valuation of life insurance contracts with guarantees challenging.

The pricing of life insurance contracts with guarantees has been a topic of research interest for many years. Brennan and Schwartz (1976, 1979) and Boyle and Schwartz (1977) made important contributions to the academic understanding of the pricing of equity-linked products and life insurance policies using the modern option price theory. According to Milvesky and Posner (2001) and Milvesky and Salisbury (2006) research, various annuities are priced under the assumption of a constant interest rate and a geometric Brownian motion to model the underlying stock price. Considering the same setting, Baueret al. (2008) extended the work of Milvesky and Salisbury (2006) by incorporating policyholder behavior. Considering stochastic interest rates, Lin and Tan (2003) and Kijima and Wong (2007) proposed a valuation approach for equity-indexed annuities. To insure that VA contracts remain profitable for the policyholder and the issuer, Bernard et al. (2014), Cui et al. (2017), Bernard and Moenig (2019) and Landriault et al. (2021) incorporated different fee structures into the pricing framework. In addition to the pricing issue associated with the financial approach, researchers have also investigated the fair valuation of equity-linked insurance with regard to the level of solvency capital required (see e.g., Hardy (2003); Barbarin and Devolder (2005) and Feng and Volkmer (2012)). Using simulation or analytical methods where financial models permit, they analyzed liabilities for equity-linked insurance contracts and determined the fair values of parameters of these contracts using risk measures. The present article applies a risk-neutral pricing approach to VAs with minimum death and minimum life guarantees. An overview of other equitylinked life insurance policies is provided by Hardy (2003) and Bacinello et al. (2011). For VAs with minimum death and life guarantees, a benefit is paid either at the time of death or upon the maturity of the contract. This benefit is capped due to a guaranteed rate and depends on the performance of the underlying investment fund. Hence, VA holders receive at least the guaranteed amount during periods of crisis and earn additional expected income in periods of economic growth due to the good performance of investment funds. In contrast, as observed during the credit crunch that occured between October 2007 and March 2009, the main VA issuers incurred large losses. These losses were explained with reference to rising guarantee values, the collapse of earnings from mutual fund fees, negative hedging results and exploding hedging costs. As VAs offer protection of capital during periods of recession and provide an additional returns in conjunction with growth to their holders, incorporating switches in economic regimes into the VAs valuation framework is thus a good way of reducing the risk of losses for VA issuers.

In the finance literature, Goldfeld and Quandt (1973) developed the regime-switching technique. Hamilton (1989) popularized this technique in economics and econometrics. In finance, regime-switching models are based on the simple and natural notion that the economic environment is not stable but subject to regular changes at certain non-predictable

\footnotetext{
1 According to the Insured Retirement Institute. https://www.myirionline.org/newsroom/newsroom-detailview/iri-issues-second-quarter-2019-annuity-sales-report
} 
stopping times. Various authors in the field of actuarial science have explored the use of regime-switching models in a continuous-time Markov switching framework. Without intending to present an exhaustive list, we refer to Hardy (2001), Siu (2005) Lin et al. (2009), Hainaut (2014), Fan et al. (2015) and Ignatieva et al. (2016). The common point of these articles is the use of diffusion processes modulated by a continuous-time Markov chain to model the interest rate and/or the reference risky asset. To the best of our knowledge, self-exciting jump-diffusion processes modulated by a continuous time hidden Markov chain have not been explored in the valuation framework of equity-linked life insurance contracts such as VAs. The literature on switching jump-diffusion is less abundant. For instance, Hainaut and Colwell (2016) extend the framework of Siu (2005) by using Markov regime-switching jump diffusion models. Fan et al. (2015) price annuity guarantees under double regime-switching models in which the underlying stock price was modulated by a Markov chain and its jump part depends on the number of state changes recorded. Hainaut and Moraux (2018) showed that the self-excitation property of the Hawkes process combined with regime-switching models enables one to better reproduce the dynamics of financial markets. In this framework, the features of shocks are modulated by economic cycles. The authors' empirical analysis of the S\&P 500, reveals that selfexcited jumps occur mainly during economic recessions and nearly disappear in periods of economic growth. The present article considers the setting of Hainaut and Moraux (2018) which integrated specific features of stock price dynamics such as jumps, heavy-tails of the return's distribution, the time-varying volatility, the regime-switching and, for option price, implied volatility smiles.

In the cited articles, the interest rates used for discounting cash flows are mostly considered constant. This assumption seems unrealistic for VAs, as potential increases in interest rates could have adverse effects on their commitments, mainly due to declining asset values. The literature features many stochastic interest rates models (e.g., Hull-White model (1990), Cox-Ingersoll-Ross (CIR) model (1985), Vasicek model (1977)) that allow for the replication of interest rate features such as time consistency, negativity, mean revertion, time-varying volatility and the clustering effect. Without losing generality, we consider a Hull-White model correlated to the stock price model. As VAs are long-term commitments, under the real measure, we fit the interest rate model using the Kalman filter (1960) to reflect the long-term trend.

This article proposes an integrated framework for the valuation of VAs that guarantee a minimum benefit in case of death or life. Our work makes four contributions to the actuarial literature. First, this work introduces self-exciting switching jump-diffusion (SESJD) models into the standard pricing framework of VAs. Second, we design a valuation framework with a stochastic interest rate model correlated to the stock price process, and from a management perspective, we consider a portfolio consisting of a bond and a risky asset as the reference investment fund. Third, we define an equivalent martingale measure and derive an approximate closed-form expression for the pricing of VAs that guarantee a minimum living and death benefit. Finally, we present the detailed procedure used for the econometric calibration of our models based on the filtering technique.

The remainder of this article is structured as follows: in Section 2, we specify the hidden Markov chain model, the SESJD model and the interest rates model. Thereafter, we define a Radon Nikodym density for our equivalent martingale pricing measure. Under this pricing measure we find the dynamics of the investment portfolio and the moment generating function of the couple formed by the cumulated short-rate and the portfolio price. We conclude this section with a pricing formula of a European call option written on the investment portfolio obtained by an inverse Fourier transform. In Section 3, we specify our VA 
contract, and based on this contract, we provide an approximate closed-form expression of its price. In Section 4, we first discuss about the calibration of our models in the real world and then present a numerical implementation of our valuation framework, focusing on the impact of VA features.

\section{The Financial Risk}

We consider a complete probability space $(\Omega, \mathcal{F}, \mathbb{P})$ equipped with a filtration $\left\{\mathcal{F}_{t}\right\}_{t>0}$ that is generated by a continuous Markov chain and other processes we specify hereafter. Here, the probability measure $\mathbb{P}$ on $(\Omega, \mathcal{F})$ denotes the real world probability measure. The time set is $\mathrm{T}=\left[0, T^{*}\right]$, where $T^{*}<\infty$ is the final trading date. We consider a financial market in which security prices are adapted to the subfiltration $\left(\mathcal{H}_{t}\right)_{t \geq 0}$. Throughout this paper, the economy is categorized into $N$ states or regimes indexed by a set of integers $\mathcal{N}:=\{1,2, \ldots, N\}$. The information about the state of economy over time, is carried by a (continuous) hidden Markov chain $\zeta(t)$ taking values from a set of unit vectors $E=\left\{e_{1}, e_{2}, \ldots, e_{N}\right\}$, where $e_{j}=(0, \ldots, 0,1,0, \ldots 0)^{\top}$ with 1 at the $j^{t h}$ position. The natural filtration generated by $\{\zeta(t)\}_{t \geq 0}$ is denoted by $\left\{\mathcal{G}_{t}\right\}_{t \geq 0}$, where $\mathcal{G}_{t}=\sigma(\zeta(s): s \in \mathrm{T}, s \leq t)$. The generator of $\zeta(t)$ is an $N \times N$ matrix $Q_{0}:=\left[q_{i, j}\right]_{i, j=1,2, \ldots, N}$, whose elements satisfy the following standard conditions:

$$
q_{i, j} \geq 0, \quad \forall i \neq j, \quad \text { and } \sum_{j=1}^{N} q_{i, j}=0, \quad \forall i \in \mathcal{N} .
$$

Each $q_{i, j}$ for $i \neq j$ is the instantaneous transition rate from state $i$ to state $j$, while $-q_{i, i}=\sum_{j \neq i, j=1}^{N} q_{i, j}>0$ is the instantaneous exit rate from state $i$. Hence,

$$
\begin{gathered}
\lim _{\Delta \rightarrow 0} \frac{P\left(\zeta(t+\Delta)=e_{j} \mid \zeta(t)=e_{i}\right)}{\Delta}=q_{i, j}, \\
\lim _{\Delta \rightarrow 0} \frac{1-P\left(\zeta(t+\Delta)=e_{i} \mid \zeta(t)=e_{i}\right)}{\Delta}=-q_{i, i} .
\end{gathered}
$$

The matrix of transition probabilities over the time interval $\lfloor t, s\rfloor$ is denoted as $P(t, s)$ and is defined by:

$$
P(t, s)=\left(p_{i, j}(t, s)\right)_{1 \leq i, j \leq N}=\exp \left(Q_{0}(s-t)\right), \quad s \geq t,
$$

where for $1 \leq i, j \leq N, p_{i, j}(t, s)$ is the probability that the chain switches from state $i$ at time $t$ to state $j$ at time $s$. Moreover, the probability that the chain is in state $i$ at time $s$ denoted by $p_{i}(s)$ depends upon the initial probabilities $p_{k}(0)$ and the transition probabilities $p_{k, i}(0, s)$, where $k=1,2, \ldots, N . p_{i}(s)$ is defined as follows:

$$
p_{i}(s)=P\left(\zeta(s)=e_{i}\right)=\sum_{k=1}^{N} p_{k}(0) p_{k, i}(0, s), \quad \text { for all } 1 \leq i \leq N .
$$

From the semi-martingale representation theorem for $\zeta(t)$ provided by Elliott et al. (2005) and given that $\mathcal{G}_{t}$ is the filtration of $\zeta(t)$, we can write $\zeta(t)$ as the sum of a $\mathcal{G}_{t}$ predictableadapted process and of a $\mathcal{G}_{t}$ martingale increment process, $\{M(t)\}_{t \geq 0}$ : 


$$
\zeta(t)=\zeta(0)+\int_{0}^{t} Q_{0}^{\top} \zeta(s) \mathrm{d} s+M(t)
$$

In this market, investors purchase three assets: cash, bonds and a stock. The price of this risky asset over time is a process $(S(t))_{t \geq 0}$ modulated by the Markov chain. We assume that the insurer invests the premium from the VA contract in a portfolio of bonds and stocks. The process $(S(t))_{t \geq 0}$ follows a modified version of the stock price model proposed by Hainaut and Moraux (2018) in which the instantaneous return is the sum of a drift, Brownian motions and a compensated jump process:

$$
\begin{aligned}
\frac{\mathrm{d} S(t)}{S\left(t^{-}\right)} & =\mu(t) \mathrm{d} t+\rho \sigma(t) \mathrm{d} W_{r}(t)+\sqrt{1-\rho^{2}} \sigma(t) \mathrm{d} W_{S}(t) \\
& +\mathrm{d}\left(\sum_{i=1}^{N(t)}\left(e^{J_{i}}-1\right)\right)-\lambda(t) \mathbb{E}\left(e^{J}-1\right) \mathrm{d} t .
\end{aligned}
$$

We assume that the instantaneous average growth rate $\mu(t)$ of the stock and the Brownian volatility $\sigma(t)$ are modulated by the Markov chain $\zeta(t)$. In detail, $\mu(t)=\zeta(t)^{\top} \bar{\mu}$ and $\sigma(t)=\zeta(t)^{\top} \bar{\sigma}$, where $\bar{\mu}=\left(\bar{\mu}_{1}, \bar{\mu}_{2}, \ldots, \bar{\mu}_{N}\right)^{\top} \in \mathbb{R}_{+}^{N}$ and $\bar{\sigma}=\left(\bar{\sigma}_{1}, \bar{\sigma}_{2}, \ldots, \bar{\sigma}_{N}\right) \mathbb{R}_{+}^{N} \cdot W_{r}(t)$ and $W_{S}(t)$ are two independent Brownian motions, and $\rho$ is the correlation coefficient between the short rate and the stock price. The amplitude of shocks $J_{i}$ are i.i.d. random double exponential variables (DEJ) with density $v(z)$ on $\mathbb{R}$. Further details about the jump size distribution and moments are provided in Appendix A. It is important to note that most of the results presented in this work are applicable to any other jump size distributions that enables both negative and positive jumps. The counting process $(N(t))_{t \geq 0}$ with intensity $(\lambda(t))_{t \geq 0}$ counts the number of observed shocks independent of their amplitude up to time $t$. To replicate the clustering of shocks observed in stocks markets, the intensity is stochastic and defined as follows :

$$
\lambda(t)=\lambda(0)-\beta \int_{0}^{t} e^{\beta(s-t)}(\lambda(0)-\eta(s)) \mathrm{d} s+\int_{0}^{t} \gamma e^{\beta(s-t)} \mathrm{d} L_{s},
$$

or in terms of stochastic differential equation (SDE) by :

$$
\mathrm{d} \lambda(t)=\beta(\eta(t)-\lambda(t)) \mathrm{d} t+\gamma \mathrm{d} L(t),
$$

where $\beta \geq 0$ is the speed of reversion, $L(t)=\sum_{i=1}^{N(t)}\left|J_{i}\right|$ enables to increase the intensity $\lambda(t)$ of $\gamma|J|$ when a jump occurs. The long-run level $\eta(t)$ is modulated by the Markov chain $\zeta(t): \eta(t)=\zeta(t)^{\top} \bar{\eta}$, where $\bar{\eta}=\left(\bar{\eta}_{1}, \bar{\eta}_{2}, \ldots, \bar{\eta}_{N}\right)^{\top} \in \mathbb{R}_{+}^{N}$. The information about this counting process is contained in the subfiltration $\mathcal{H}$. Hainaut and Moraux (2018) derive from their proposition 2.1 that conditionally to $\mathcal{H}_{0}$ the expectation of $\lambda(t)$ converges when $t$ tends to infinity only if the speed of mean reversion is larger than $\gamma \mathbb{E}(|J|)$. In the calibration section, this stability condition ensures that the number of shocks hitting the stock price process $S(t)$ does not explode as the maturity increases.

To preserve the analytical tractability, we assume that the risk-free rate $(r(t))_{t \geq 0}$ is not modulated by the Markov chain and satisfies, under $\mathbb{P}$ this SDE:

$$
\mathrm{d} r(t)=\left(\theta^{\mathbb{P}}(t)-a_{r} r(t)\right) \mathrm{d} t+\sigma_{r} \mathrm{~d} W_{r}(t),
$$

where the level of mean reversion $\theta^{\mathbb{P}}(t)$ is a process, $a_{r} \geq 0$ is the speed of mean-reversion and $\sigma_{r} \geq 0$ is the volatility of the interest rates market. In order to price bonds, we define a risk neutral measure. However, the market is incomplete due to regime switches and jumps. 
The risk-neutral measure derived under the martingale condition is not unique, which is also the case with the price of a given product in this market. Different equivalent measures such as the Esscher transform measure, the minimal entropy measure or the varianceoptimal measure, could be used, but, in this paper, we do not discuss the selection criteria of an equivalent martingale measure. Instead, we consider that the equivalent martingale measure $\mathbb{Q}$ of $\mathbb{P}$ with the Radon Nykodym density defined by:

$$
\begin{aligned}
\Lambda(t)=\left.\frac{\mathrm{d} \mathbb{Q}}{\mathrm{d} \mathbb{P}}\right|_{\mathcal{F}_{t}} & =: \exp \left(-\frac{1}{2} \int_{0}^{t}\left(\sigma_{r}^{2} \theta_{r}^{2}+\sigma^{2}(u) \theta_{S}^{2}(u)\right) \mathrm{d} u\right. \\
& \left.-\int_{0}^{t} \sigma_{r} \theta_{r} \mathrm{~d} W_{r}(u)-\int_{0}^{t} \sigma(u) \theta_{S}(u) \mathrm{d} W_{S}(u)\right)
\end{aligned}
$$

where $\theta_{S}(t)=\zeta(t)^{\top} \bar{\theta}_{S}, \theta_{r} \in \mathbb{R}$ and $\bar{\theta}_{S} \in \mathbb{R}^{N}$. Using the Itô's differentiation rule, we find that $\mathrm{d} \Lambda(t)=-\sigma_{r} \theta_{r} \mathrm{~d} W_{r}(t)-\sigma(t) \theta_{S}(t) \mathrm{d} W_{S}(t)$. Then, as $\Lambda(t)$ is an $\mathcal{F}$-martingale under the probability measure $\mathbb{P}$ and $\mathbb{E}(\Lambda(t))=1$, our equivalent measure $\mathbb{Q}$ is well-defined. If $\theta_{r}$ is a constant and $\theta_{S}(t)$ is such that

$$
\theta_{S}(t)=\frac{\mu(t)-r(t)-\rho \sigma_{r} \theta_{r} \sigma(t)}{\sqrt{1-\rho^{2}} \sigma^{2}(t)},
$$

then the discounted price process of the risky asset $\exp \left(-\int_{0}^{t} r(u) \mathrm{d} u\right) S(t)$ is a $\mathcal{F}$-martingale under the probability measure $\mathbb{Q}$. Under this risk neutral measure, $(S(t))_{t \in \mathrm{T}}$ and $(r(t))_{t \in \mathrm{T}}$ satisfy the following SDEs :

$$
\begin{gathered}
\mathrm{d} r(t)=\left(\theta^{\mathbb{Q}}(t)-a_{r} r(t)\right) \mathrm{d} t+\sigma_{r} \mathrm{~d} W_{r}^{\mathbb{Q}}(t), \\
\frac{\mathrm{d} S(t)}{S\left(t^{-}\right)}=r(t) \mathrm{d} t+\rho \sigma(t) \mathrm{d} W_{r}^{\mathbb{Q}}(t)+\sqrt{1-\rho^{2}} \sigma(t) \mathrm{d} W_{S}^{\mathbb{Q}}(t) \\
+\mathrm{d}\left(\sum_{i=1}^{N(t)}\left(e^{J_{i}}-1\right)\right)-\lambda(t) \mathbb{E}\left(e^{J}-1\right) \mathrm{d} t,
\end{gathered}
$$

where the processes $\left(W_{r}^{\mathbb{Q}}(t)\right)_{t \in \mathrm{T}}$ and $\left(W_{S}^{\mathbb{Q}}(t)\right)_{t \in \mathrm{T}}$ are two Brownian motions under $\mathbb{Q}$ such that:

$$
\begin{aligned}
& W_{r}^{\mathbb{Q}}(t)=W_{r}(t)+\sigma_{r} \theta_{r} t, \\
& W_{S}^{\mathbb{Q}}(t)=W_{S}(t)+\int_{0}^{t} \sigma(u) \theta_{S}(u) \mathrm{d} u .
\end{aligned}
$$

$\theta^{\mathbb{Q}}(t)=\theta^{\mathbb{P}}(t)-\sigma_{r}^{2} \theta_{r}$ is a deterministic function of the time, where $-\sigma_{r} \theta_{r}$ is the interest rate's risk price. We note that the market price of risk $-\theta_{S}(t) \sigma(t)$ in our framework depends on the state of the economy and the instantaneous short rate. For the sake of simplicity, we assume that the distribution of the hidden Markov chain $\zeta$ and the modulated jump process $(\lambda, N)$ remain unchanged after the measure change from $\mathbb{P}$ to $\mathbb{Q}$. Thus, the semimartingale representation of the hidden Markov chain in Equation (1) still holds.

In this setting, asset prices are valued as the sum of their expected discounted cash flows under the risk-neutral measure. From Equation (7), the short-rate follows a HullWhite process (1990) under $\mathbb{Q}$. The price of a zero-coupon bond at time $t$ with maturity $T$ is given by: 


$$
Z C(t, T)=\mathbb{E}^{\mathbb{Q}}\left[e^{-\int_{t}^{T} r(s) \mathrm{d} s} \mid \mathcal{F}_{t}\right]=e^{-A^{r}(t, T)-B^{r}(t, T) r(t)},
$$

where

$$
B^{r}(t, T)=\frac{1}{a_{r}}\left(1-e^{-a_{r}(T-t)}\right),
$$

and

$$
A^{r}(t, T)=\int_{t}^{T} f^{M}(0, s) \mathrm{d} s-f^{M}(0, t) B^{r}(t, T)+\frac{\sigma_{r}^{2}}{4 a_{r}}\left(1-e^{-2 a_{r} t}\right) B^{r}(t, T)^{2},
$$

with $f^{M}(0, t)$ the instantaneous forward rate observed at time 0. From Equation (9), we have the SDE of this zero coupon bond :

$$
\frac{\mathrm{d} Z C(t, T)}{Z C\left(t^{-}, T\right)}=r(t) \mathrm{d} t-\sigma_{r} B^{r}(t, T) \mathrm{d} W_{r}^{\mathbb{Q}}(t) .
$$

It follows that $-\theta_{r} \sigma_{r}^{2} B^{r}(t, T)$ represents the instantaneous risk premium for an investment in such a zero-coupon bond.

The equations obtained under the measure $\mathbb{Q}$ are used later to value the portfolio in which the insurer invests the unique premium. Composed of a zero coupon bond and a risky asset, the value of this portfolio denoted by $V(t)$ at time $t$ is defined as follows :

$$
\frac{\mathrm{d} V(t)}{V\left(t^{-}\right)}=\omega_{S} \frac{\mathrm{d} S(t)}{S\left(t^{-}\right)}+\left(1-\omega_{S}\right) \frac{\mathrm{d} Z C(t, T)}{Z C\left(t^{-}, T\right)},
$$

where $\omega_{S}$ is the proportion of the premium invested in the risky asset. Without loss of generality, we assume that our portfolio is self-financed and continuously re-balanced in order to keep this proportion constant over the time. Under the risk neutral measure from Equation (13) the $\log$-return of the investment portfolio $X(t):=\ln \frac{V(t)}{V(0)}$ satisfies the following SDE :

$$
\begin{aligned}
\mathrm{d} X(t)= & \left(r(t)+\omega_{S}\left(1-\omega_{S}\right) \rho \sigma(t) \sigma_{r} B^{r}(t, T)-\frac{1}{2} \omega_{S}^{2} \sigma^{2}(t)\right) \mathrm{d} t \\
& -\left(\frac{1}{2}\left(1-\omega_{S}\right)^{2} \sigma_{r}^{2}\left(B^{r}(t, T)\right)^{2}+\omega_{S} \lambda(t) \mathbb{E}\left(e^{J}-1\right)\right) \mathrm{d} t \\
+ & \left(\omega_{S} \rho \sigma(t)-\left(1-\omega_{S}\right) \sigma_{r} B^{r}(t, T)\right) \mathrm{d} W_{r}^{\mathbb{Q}}(t) \\
& +\omega_{S} \sqrt{1-\rho^{2}} \sigma(t) \mathrm{d} W_{S}^{\mathbb{Q}}(t)+\ln \left(1+\omega_{S}\left(e^{J}-1\right)\right) \mathrm{d} N(t) .
\end{aligned}
$$

We conclude this section with a proposition used to price our VA contract. To price embedded options in the VA, we need the moment generating function (mgf) of the bivariate random variable $\left(\int_{t}^{s} r(u) \mathrm{d} u, X(s)\right)$ given the filtration $\mathcal{F}_{t}$ with $t \leq s$.

Proposition 1 The $m g f M_{r, X}^{t, s}$ of the pair of random variables $\left(\int_{t}^{s} r(u) \mathrm{d} u, X(s)\right)$ given the filtration $\mathcal{F}_{t}$ with $\bar{\omega}=\left(\omega_{1}, \omega_{2}\right) \in \mathbb{C}_{-}^{2}$ and $t \leq s$ is equal to : 


$$
\begin{aligned}
M_{r, X}^{t, s}\left(\omega_{1}, \omega_{2}\right) & =\mathbb{E}^{\mathbb{Q}}\left(e^{\omega_{1} \int_{t}^{s} r(u) \mathrm{d} u+\omega_{2} X(s)} \mid \mathcal{F}_{t}\right) \\
& =\left(\frac{V(t)}{V(0)}\right)^{\omega_{2}} \zeta^{\top}(t) \tilde{A}(\bar{\omega}, t, s) \\
& \times \exp \left(\left(\omega_{2}+\omega_{1}\right) B^{r}(t, s) r(t)+C(\bar{\omega}, t, s) \lambda(t)\right),
\end{aligned}
$$

where $\tilde{A}(\cdot)$ is a vector of $N$ functions, and the function $C(\cdot)$ satisfy the terminal value problems' partial differential equations (PDEs) system:

$$
\left\{\begin{array}{l}
0=\frac{\partial \tilde{A}(\bar{\omega}, t, s)}{\partial t}+\left(\operatorname{diag}(D(\bar{\omega}, t, s))+Q_{0}\right) \tilde{A}(\bar{\omega}, t, s) \\
0=C_{t}-\omega_{2} \omega_{S} \mathbb{E}^{\mathbb{Q}}\left(e^{J}-1\right)-\beta C+\mathbb{E}^{\mathbb{Q}}(g(J, C))-1
\end{array}\right.
$$

with $g:(x, C) \longmapsto e^{\omega_{2} \ln \left(1+\omega_{S}\left(e^{x}-1\right)\right)+C \gamma|x|}$, a real valued bivariate function,

$$
\begin{gathered}
D(\bar{\omega}, t, s)=-\frac{\omega_{2}}{2}\left(1-\omega_{2}\right)\left(D_{1}^{2}+\omega_{S}^{2}\left(1-\rho^{2}\right) \bar{\sigma}^{2}\right)+\theta^{\mathbb{Q}}(t) D_{2}+\frac{1}{2} \sigma_{r}^{2} D_{2}^{2} \\
+\omega_{2} \sigma_{r} D_{1} D_{2}+\beta \bar{\eta} C, \\
D_{1}=\omega_{S} \rho \bar{\sigma}-\left(1-\omega_{S}\right) \sigma_{r} B^{r}(t, T), \quad D_{2}=\left(\omega_{2}+\omega_{1}\right) B^{r}(t, s), \quad C(\bar{\omega}, s, s)=0 \quad \text { and }
\end{gathered}
$$
$\tilde{A}(\bar{\omega}, s, s)=1_{\mathbb{R}^{N}}$.

\section{Proof In Appendix B}

Note that while the PDE system (16) does not admit an analytical solution, a numerical solutions can be found using a numerical method. The joint distribution of $\left(\int_{t}^{s} r(u) \mathrm{d} u, X(s)\right)$ defined by this mgf allows us to price by Fourier transform inversion, a European put option written on the portfolio value $V$ with maturity $T$ and strike $V(t) e^{k}$. Let $p_{T}(k)$ be the value of this European put option and $\psi$ the joint density of $\left(\int_{t}^{s} r(u) \mathrm{d} u, X(s)\right)$ under $\mathbb{Q}$. Thus,

$$
p_{T}(k)=V(t) \int_{-\infty}^{k} \int_{-\infty}^{+\infty} e^{-y_{1}}\left(e^{k}-e^{y_{2}}\right) \psi\left(y_{1}, y_{2}\right) \mathrm{d} y_{1} \mathrm{~d} y_{2} .
$$

This European put option is not square integrable since $p_{T}$ tends to infinity instead of zero when the $\log$ strike $k$ tends to $+\infty$. To obtain a square integrable function, we consider the modified put option price:

$$
\bar{p}_{T}(k):=\exp (-\alpha k) p_{T}(k)
$$

where $\alpha>0$ is chosen such that $\bar{p}_{T}$ is a square-integrable function. Then the Fourier transform of $\bar{p}_{T}$ exists and is defined by

$$
\mathcal{F}\left(\bar{p}_{T}\right)(v)=\int_{-\infty}^{+\infty} e^{i v k} \bar{p}_{T}(k) \mathrm{d} k,
$$

and, by inverse Fourier transform, the option price is obtained numerically using the following equation :

$$
p_{T}(k)=\frac{e^{\alpha k}}{\pi} \int_{0}^{+\infty} e^{-i v k} \mathcal{F}\left(\bar{p}_{T}\right)(v) \mathrm{d} v .
$$


Applying Simpson's rule to the integral in Equation (20), we approximate put options prices in the following proposition:

Proposition 2 Let $M$ be the number of steps used in the discrete Fourier transform and $\Delta_{k}=\frac{2 k_{\max }}{M}$ the step of discretization. Let us denote $\delta_{j}=\frac{1}{3}\left(3+(-1)^{j}-1_{\{j=1\}}\right), \Delta_{v}=\frac{2 \pi}{M \Delta_{k}}$ and $v_{j}=(j-1) \Delta_{v}$. The value of $p_{T}(k)$ at points $k_{j}=-k m a x+(j-1) \Delta_{k}$ is approximated by

$$
p_{T}\left(k_{j}\right) \approx \frac{2 V(t) e^{\alpha k_{j}}}{M \Delta_{k}} \sum_{l=1}^{M} \delta_{l} e^{-\frac{2 \pi i}{M}(j-1)(l-1)}(-1)^{l-1} \frac{M_{r, X}^{t, T}\left(-1,1-\alpha+i v_{l}\right)}{\alpha^{2}-\alpha-v_{l}^{2}+i(-2 \alpha+1) v_{l}} .
$$

for all $j=1, \ldots, M$ and any $\alpha>0$ such that $M_{r, X}^{t, T}(-1,1-\alpha)<+\infty$. This last relation can be computed with a fast Fourier transform algorithm.

\section{Proof In Appendix D}

In the next section, we consider several of the different forms of VA contract and price them within our modelling framework.

\section{Valuation of the Variable Annuity}

\subsection{Definitions}

The VA contract valued under our models setting consists of a guaranteed minimum accumulation benefit (GMAB) and guaranteed minimum death benefit (GMDB). Any holder of this VA contract pays a unique premium $\Pi>0$ in exchange for benefits that depend on the return of the investment portfolio. The policy charges fees for management expenses such as the mortality fee, administrative costs, management fees and other fees linked to the contract riders. These fees are charged annually to the account value of the VA contract. If we denote by $\varphi$ the proportional fee rate applied to the account value in order to recover the cost of all the options, then the account value $A$ satisfies the following SDE:

$$
\frac{\mathrm{d} A(t)}{A\left(t^{-}\right)}=\frac{\mathrm{d} V(t)}{V\left(t^{-}\right)}-\varphi \mathrm{d} t,
$$

where its initial value $A(0)$ is equal to the unique premium $\Pi$. Let $\delta \geq 0$ be the guaranteed interest rate used to reassess the premium during the lifetime of the VA contract. Our VA contract provides a minimum guaranteed benefit in case of death and a lump sum payment upon expiry. If withdrawal and surrender are not allowed during the contract's lifetime, benefits are defined as follows

- GMDB: In case of death prior to the stated maturity $T$, the insurer pays the maximum between the account value and a guaranteed amount. At the death time $\tau_{d}, t<\tau_{d} \leq T$, the benefit is $b_{D}\left(\tau_{d}\right)=\max \left\{A\left(\tau_{d}\right), G\left(\tau_{d}\right)\right\}$, where $G(0) \in(0, \Pi]$ and the common guaranteed amount $G\left(\tau_{d}\right)$ on the market is

- either the roll-up of the premium at the guaranteed interest rate, called the roll-up guaranteed 


$$
G\left(\tau_{d}\right)=G(t) e^{\delta\left(\tau_{d}-t\right)},
$$

- or the highest account value recorded every year before the death, called the ratchet guaranteed,

$$
G\left(\tau_{d}\right)=\max _{\left.j \in\left\{1, \cdots, \tau_{d}\right\rfloor\right\}}(G(t), A(t+j)) .
$$

- GMAB: At expiry date $T$ (typically the end of the accumulation period), the insured, if alive, receives the maximum between the policy account value and the guaranteed amount. The benefit is

$$
b_{L}(T)=\max \{A(T), G(T)\}
$$

where the guaranteed amount $G(T)$ is expressed similarly as in the death case 24 . The fair proportional fee rate $\varphi$ is the solution of the following equation:

$$
\Pi:=L(t, T, \varphi),
$$

where $L(t, T, \varphi)$ denotes the total liabilities of the insurer at time $t$. This total liability is the net of the proportional fee $\varphi$ which is deducted from the investment fund value.

\subsection{Valuation}

The theorem of asset pricing and the linearity of the expectation allow us to define the total liabilities of the insurer at time $t$ toward an insured aged $x$ at time $t$ as follows:

$$
\begin{aligned}
L(t, T, \varphi) & :=\mathbb{E}^{\mathbb{Q}}\left(e^{-\int_{t}^{\tau_{d}} r(u) \mathrm{d} u} 1_{\left\{\tau_{d}<T\right\}} b_{D}\left(\tau_{d}\right)+e^{-\int_{t}^{T} r(u) \mathrm{d} u} 1_{\left\{\tau_{d}>T\right\}} b_{L}(T)\right) \\
& =L_{D}(t, T, \varphi)+L_{L}(t, T, \varphi),
\end{aligned}
$$

where $t$ and $T$ are integers. $L_{D}(t, T, \varphi)$ and $L_{L}(t, T, \varphi)$ are respectively the present values of the death benefit and of the survival benefit at time $t$. These quantities are evaluated under the assumption that mortality risk is independent from financial risks. We also assume that individuals die at the end of the year and that the death benefit is paid at the end of the death year. This latter assumption is arguable but can be adapted according to the terms of the contract. The death liability in Equation (27) is then given by:

$$
\begin{aligned}
L_{D}(t, T, \varphi) & =\mathbb{E}^{\mathbb{Q}}\left(e^{-\int_{t}^{\tau_{d}} r(u) \mathrm{d} u} 1_{\left\{\tau_{d}<T\right\}} b_{D}\left(\tau_{d}\right) \mid \mathcal{F}_{t}\right) \\
& =\sum_{j=0}^{T-t-1}{ }_{j} p_{x+t} q_{x+t+j} \mathbb{E}^{\mathbb{Q}}\left(e^{-\int_{t}^{t+j+1} r(u) \mathrm{d} u} b_{D}(t+j+1) \mid \mathcal{F}_{t}\right),
\end{aligned}
$$

where $t+1, t+2, \ldots, T$ are the possible dates of payment of death benefits until the maturity $T$, whereas the life liability $L_{L}(t, T, \varphi)$, is given by:

$$
\begin{aligned}
L_{L}(t, T, \varphi) & =\mathbb{E}^{\mathbb{Q}}\left(e^{-\int_{t}^{T} r(u) \mathrm{d} u} 1_{\left\{\tau_{d}>T\right\}} b_{L}(T) \mid \mathcal{F}_{t}\right) \\
& ={ }_{T} p_{x+t} \mathbb{E}^{\mathbb{Q}}\left(e^{-\int_{t}^{T} r(u) \mathrm{d} u} b_{L}(T) \mid \mathcal{F}_{t}\right),
\end{aligned}
$$


where the life benefit $b_{L}(T)=\max \{A(T), G(T)\}$ changes according to the two type of the guaranteed amount considered hereafter:

- Roll-up guaranteed In this case, the guaranteed amount $G(T)$ satisfies Equation (23) and the life benefit is the maximum between a random variable and a constant. This life benefit can be rewritten as a function of the payoff of a put option and enables us to simplify the life liability formula as follows:

$$
\begin{gathered}
L_{L}(t, T, \varphi)={ }_{T} p_{x+t} \mathbb{E}^{\mathbb{Q}}\left(e^{-\int_{t}^{T} r(u) \mathrm{d} u} \max \left\{A(T), G(t) e^{\delta(T-t)}\right\} \mid \mathcal{F}_{t}\right) \\
={ }_{T} p_{x+t} \mathbb{E}^{\mathbb{Q}}\left(e ^ { - \int _ { t } ^ { T } r ( u ) \mathrm { d } u } \left(A(t) e^{X(T)-X(t)-\varphi(T-t)}\right.\right. \\
\left.\left.+\left(G(t) e^{\delta(T-t)}-A(t) e^{X(T)-X(t)-\varphi(T-t)}\right)_{+}\right) \mid \mathcal{F}_{t}\right) \\
={ }_{T} p_{x+t} \mathbb{E}^{\mathbb{Q}}\left(e ^ { - \int _ { t } ^ { T } r ( u ) \mathrm { d } u } \left(A(0) e^{X(T)-\varphi T}\right.\right. \\
\left.\left.\quad+\left(G(0) e^{\delta T}-A(0) e^{X(T)-\varphi T}\right)_{+}\right) \mid \mathcal{F}_{t}\right) \\
={ }_{T} p_{x+t} e^{-\varphi T} A(0)\left(\mathbb{E}^{\mathbb{Q}}\left(e^{-\int_{t}^{T} r(u) \mathrm{d} u+X(T)} \mid \mathcal{F}_{t}\right)\right. \\
\left.+\mathbb{E}^{\mathbb{Q}}\left(e^{-\int_{t}^{T} r(u) \mathrm{d} u}\left(\frac{G(0)}{A(0)} e^{(\varphi+\delta) T}-e^{X(T)}\right) \mid \mathcal{F}_{t}\right)\right) \\
={ }_{T} p_{x+t} A(0) e^{-\varphi T}\left(M_{r, X}^{t, T}(-1,1)+p_{T}(t)\right) .
\end{gathered}
$$

where $p_{T}(t)$ is the price of a European put option with maturity $T$ and strike $\frac{G(0)}{A(0)} e^{(\delta+\varphi) T}$. Recall that the initial account value $A(0)$ equals $\Pi$ and the initial amount guaranteed $G(0)$ is in the interval $(0, \Pi]$. From Equation $(31)$, the insurer commitment of a GMAB is then hedgeable by investing in cash and a put option.

- Annual ratchet guaranteed The life benefit is the highest account value $A$ recorded every year until the maturity $T$. Since the account value at any time depends on the state of the chain, the property of the independence of increments does not hold. While we can not deduce a closed-form expression in this case, the life liability for a guaranteed annual ratchet:

$$
\begin{aligned}
L_{L}(t, T, \varphi) & ={ }_{T} p_{x+t} \mathbb{E}^{\mathbb{Q}}\left(e^{-\int_{t}^{T} r(u) \mathrm{d} u}\right. \\
& \left.\times \max \left\{A(T), \max _{j \in\{1, \cdots, T-t-1\}}\{G(t), A(t+j)\}\right\} \mid \mathcal{F}_{t}\right)
\end{aligned}
$$

can be evaluated using the Monte Carlo approach.

In addition to the life liability, the death liability 28 can be rewrite as a weighted sum of life commitments in this way: 


$$
\begin{aligned}
L_{D}(t, T, \varphi) & =\sum_{j=0}^{T-t-1}{ }_{j} p_{x+t} q_{x+j} \frac{{ }_{j+1} p_{x+1}}{{ }_{x+t}} \mathbb{E}^{\mathbb{Q}}\left(e^{-\int_{t}^{t+j+1}\left(r_{u}+v_{u}\right) \mathrm{d} u} b_{L}(t+j+1) \mid \mathcal{F}_{t}\right) \\
& =\sum_{j=0}^{T-t-1}\left(\frac{{ }_{j} p_{x+t}}{{ }_{j+1} p_{x+t}}-1\right) L_{L}(t, t+j+1, \varphi) .
\end{aligned}
$$

It follows that for a roll-up guaranteed, the death liability

$$
\begin{aligned}
L_{D}(t, T, \varphi)= & A(0) \sum_{j=0}^{T-t-1}\left(\frac{{ }_{j} p_{x+t}}{{ }_{j+1} p_{x+t}}-1\right){ }_{t+j+1} p_{x+t} e^{-\varphi(t+j+1)} \\
& \times\left(M_{r, X}^{t, t+j+1}(-1,1)+p_{t+j+1}(t)\right)
\end{aligned}
$$

is also hedgeable by investing in cash and in put options with different maturities.

\section{Numerical Emplementation}

This section is divided into four subsections. The first subsection presents the mortality rate model and its estimated parameters, while the second subsection details the econometric calibration of our interest rate model via the Kalman filter approach. The third subsection presents the estimation procedure of our stock models when the hidden Markov chain $\zeta$ has three states: $e_{1}$ for "boom" or "trend up", $e_{2}$ for "recession" or "trend down" and $e_{3}$ for "crisis" or "trend sidway". Finally, we perform a sensitivity analysis to highlight the impact of self-exciting jumps and economic regimes on the valuation of the VA defined in the previous section.

\subsection{Estimation of the Mortality Rate}

The mortality rate model follows the Gompertz Makeham distribution (Gompertz (2003), Makeham (1860)). This setting enables one to derive a closed-form expression of the survival probability. For an individual aged $x$ at time zero, the mortality rate $\mu(x)$ is given by:

$$
\mu(x)=a_{\mu}+b_{\mu} c_{\mu}^{x}, a_{\mu}=-\ln \left(s_{\mu}\right), \quad b_{\mu}=-\ln \left(g_{\mu}\right) \ln \left(c_{\mu}^{x}\right),
$$

where $a_{\mu}$ is the mortality rate due to accidents and $b_{\mu} c_{\mu}^{x}$ is the term related to the ageing. The chosen parameters in Table 1 maximize the likelihood function when we consider the

Table 1 The Gompertz Makeham parameters for both sexes Belgian mortality table, obtained by likelihood maximization

\begin{tabular}{ll}
\hline$s_{\mu}$ & 0.9995465 \\
$g_{\mu}$ & 0.9998611 \\
$c_{\mu}$ & 1.106383 \\
\hline
\end{tabular}


Table 2 Descriptive statistics of the yield bonds in percent, from September 6, 2004, to April 16, 2020

\begin{tabular}{|c|c|c|c|c|c|c|c|c|c|c|}
\hline Maturity & 1 & 2 & 3 & 4 & 5 & 6 & 7 & 8 & 9 & 10 \\
\hline Mean & 0.84 & 0.97 & 1.13 & 1.31 & 1.48 & 1.65 & 1.81 & 1.95 & 2.08 & 2.19 \\
\hline Std. dev. & 1.59 & 1.61 & 1.63 & 1.62 & 1.61 & 1.61 & 1.6 & 1.59 & 1.59 & 1.58 \\
\hline Min & -0.91 & -0.97 & -1 & -1.01 & -0.996 & -0.97 & -0.93 & -0.89 & -0.87 & -0.82 \\
\hline $\operatorname{Max}$ & 4.53 & 4.71 & 4.73 & 4.73 & 4.73 & 4.72 & 4.73 & 4.74 & 4.76 & 4.78 \\
\hline
\end{tabular}

mortality tables for both sexes provided by the Belgian regulator. ${ }^{2}$ The national regulator for life annuities and death products recommends these mortality tables as a baseline.

If $\tau_{d}$ denotes the death time, we evaluate the survival probability of an individual aged $x$ at time zero as follows :

$$
{ }_{\epsilon} p_{x}=P\left(\tau_{d}>\epsilon\right)=e^{-\int_{0}^{\epsilon} \mu(x+u) \mathrm{d} u}, \forall \epsilon \geq 0 .
$$

\subsection{Econometric Calibration of the Short Rates Model}

As VAs are long-term commitments, we fit the interest rate model under the real measure $\mathbb{P}$ using the Kalman filter (1960) to reflect the long-term trend. The speed of mean reversion and volatility found in this method are next used under the risk neutral condition. We believe these parameters better reflect the long-term trend than those we could obtain by fitting the model to, for example swaptions. Our estimation procedure is divided into two steps and based on a time series of daily ECB yield bonds ${ }^{3}$ data from September 6, 2004, to April 16, 2020 for 10 maturities. Instead of using the overnight rate as a proxy for the short-term rate, in the first step we use the Kalman filter approach (1960) to guess its dynamics for the time series of daily ECB bond yields. Babbs and Nowman (1999) adopted a similar approach. Let $\left\{t_{1}, \cdots, t_{3991}\right\}$ be the set of times at which we observe these yield bonds curves. Table 2 summarizes the descriptive statistics of the yield bonds.

The bond price $Z C(t, T)$ (9) is the exponential of an affine function in $r(t)$ : $-A^{r}(t, T)-r(t) B^{r}(t, T)$, where functions $A^{r}$ and $B^{r}$ are defined in Equation (11) and (10) respectively. Therefore, the observable spot rate or yield bond $R(t, T)=-\frac{\ln (Z C(t, T))}{T-t}$ is an affine function. We consider a linear Kalman filter. The measurement equation is defined as follows :

$$
R\left(t_{k}\right)=z\left(t_{k}\right) r\left(t_{k}\right)+d\left(t_{k}\right)+\epsilon\left(t_{k}\right) \quad \epsilon\left(t_{k}\right) \sim N(0, H)
$$

where $R\left(t_{k}\right)=\left(R\left(t_{k}, t_{k}+\tau_{i}\right)\right)_{1 \leq i \leq 10}$ is the vector of yield bond for different maturities, and $z\left(t_{k}\right)=\left(\frac{B^{\prime}\left(t_{k}, t_{k}+\tau_{i}\right)}{\tau_{i}}\right)_{1 \leq i \leq 10}$ and $d\left(t_{k}\right)=\left(\frac{A^{r}\left(t_{k}, t_{k}+\tau_{i}\right)}{\tau_{i}}\right)_{1 \leq i \leq 10}$ are vectors derived from the price of zero-coupon bonds. $\tau_{i} \in\{1,2, \ldots, 10\}$ is the maturity of the considered bond. The $\epsilon\left(t_{k}\right)$ are the measurement errors assumed normally distributed with a covariance matrix $H=\operatorname{diag}\left(h_{1}, \cdots, h_{10}\right)$. Note that the variance of the measurement errors depends on

\footnotetext{
2 The Belgian mortality tables for both sexes are available via the following link http://www.ejustice.just. fgov.be/eli/arrete/2013/01/29/2013011073/justel

3 Source: http://sdw.ecb.europa.eu/browse $\cdot$ do?node $=9691126$
} 
maturities. For a step size $\Delta=\frac{1}{365}$, we have 3991 values of bond yields for each maturity $\tau_{i}$. Moreover, the transition equation of the state variable $r$ according to this step size is given by

$$
r\left(t_{k}\right)=f\left(t_{k}\right) r\left(t_{k-1}\right)+g\left(t_{k}\right)+w\left(t_{k}\right)
$$

where

$$
\begin{aligned}
f\left(t_{k}\right) & =e^{-a_{r} \Delta}, \\
g\left(t_{k}\right) & =\theta^{\mathbb{P}} B^{r}\left(t_{k-1}, t_{k}\right), \\
w\left(t_{k}\right) & \sim N\left(0, \frac{\sigma_{r}^{2}}{2 a_{r}}\left(1-e^{-2 a_{r} \Delta}\right)\right) .
\end{aligned}
$$

Let us define by $\Theta=\left\{a_{r}, \sigma_{r}, \theta_{r}, \theta^{\mathbb{P}}, h_{1}, \cdots, h_{10}\right\}$ the set of parameters of the short-rate model including the parameter from the distribution of measurement errors. We assume that error terms of the measurement and the transition equations are not correlated. The distribution of $r\left(t_{k}\right)$ then depends only on the value of $r\left(t_{k-1}\right)$, while the distribution of $R\left(t_{k}\right)$ depends only on $r\left(t_{k-1}\right)$. With the Kalman filter, we can guess the unobservable values $r\left(t_{k}\right)$ and estimate the filtering error. The likelihood function of the log likelihood for the observations $R\left(t_{1}\right), \cdots, R\left(t_{3991}\right)$ is

$$
\begin{aligned}
l_{3991} & :=\ln L\left(R\left(t_{1}\right), \cdots, R\left(t_{3991}\right) ; \Theta\right) \\
& =\ln p\left(R\left(t_{1}\right)\right)+\sum_{k=2}^{3991} \ln p\left(R\left(t_{k}\right) \mid \mathcal{H}_{t_{k-1}}\right),
\end{aligned}
$$

where $p\left(R\left(t_{k}\right) \mid \mathcal{H}_{t_{k-1}}\right)$ is the conditional distribution of $R\left(t_{k}\right)$ given the information set, $\mathcal{H}$ at time $t_{k-1} \cdot p\left(R\left(t_{1}\right)\right)$ is the initial distribution of $R\left(t_{1}\right)$ assumed as the stationary distribution of the instantaneous spot rate $r\left(t_{1}\right) \sim N\left(f\left(t_{1}\right) r(0)+g\left(t_{1}\right), \frac{\sigma_{r}^{2}}{2 a_{r}}\right)$. We find an estimate $\hat{\Theta}$ of $\Theta$ by maximizing the log likelihood in Equation (38).

In the second step, we estimate the mean reversion level $\theta^{\mathbb{Q}}$ in the short-rate model such that we fit the initial forward curve. For this purpose, we assume that the initial forward curve follows a Nelson-Siegel parametric curve (1987). The Nelson-Siegel model is a parsimonious model of yield curves widely used in practice to replicate at best the initial term structure of interest rates. Thus, we define the initial forward rate as follows:

$$
f^{M}(0, s)=z_{1}+z_{2} e^{-\alpha s}+z_{3} s e^{-\alpha s}, \quad s>0
$$

where $z_{1}, z_{2}, z_{3}$ and $\alpha>0$ are fixed real numbers. Next, we deduce a closed form of the moving target at time $s>0$ :

$$
\begin{aligned}
\theta^{\mathbb{Q}}(s)= & a_{r} z_{1}+\frac{\sigma_{r}^{2}}{2 a_{r}}+\left(z_{3}+\left(a_{r}-\alpha\right) z_{2}\right) e^{-\alpha s}+\left(a_{r}-\alpha\right) z_{3} s e^{-\alpha s} \\
& -\frac{\sigma_{r}^{2}}{2 a_{r}} e^{-2 a_{r} s} .
\end{aligned}
$$

Given the estimated values of $a_{r}, \sigma_{r}$ and $\theta_{r}$, we find $z_{1}, z_{2}, z_{3}$ and $\alpha>0$ that minimize the mean square error between the observed yield curve as of April 16, 2020, and the model yield curve where the mean reversion level satisfies Equation (40). 
Table 3 In the left side, the estimated parameters of the short-rate model. In the right side, the estimated variance of the measurement errors

\begin{tabular}{llll}
\hline$a_{r}$ & $1.61 \%$ & $\theta_{r}$ & $5.06 \%$ \\
$\sigma_{r}$ & $0.69 \%$ & $r_{0}$ & $-0.65 \%$ \\
$z_{1}$ & $-1.07 \%$ & $z_{2}$ & $0.42 \%$ \\
$z_{3}$ & $-0.75 \%$ & $\alpha$ & 1.51 \\
$h_{1}$ & $0.33 \%$ & $h_{2}$ & $0.15 \%$ \\
$h_{3}$ & $0 \%$ & $h_{4}$ & $0.13 \%$ \\
$h_{5}$ & $0.23 \%$ & $h_{6}$ & $0.32 \%$ \\
$h_{7}$ & $0.39 \%$ & $h_{8}$ & $0.45 \%$ \\
$h_{9}$ & $0.51 \%$ & $h_{10}$ & $0.55 \%$ \\
\hline
\end{tabular}

Table 3 contains estimated parameters of the short-rate model and the estimated variances of measurement errors per maturity based on the estimation procedure described previously.

Figure 1 compares the observed yield curve as of April 16, 2020, to the yield curve obtained from the Hull-White model where the initial forward curve follows a NelsonSiegel model with the estimated parameters in Table 3.

\subsection{Econometric Calibration of the Stock Price Model}

This subsection describes the procedure for estimating the SESJD model from a time series. This estimation procedure is divided into three steps. In the first step, an estimation of the switching-diffusion part is done with a Hamilton filter. The second and third steps involve detecting jumps with the peak over threshold (POT) and estimating parameters of the switching Hawkes intensity $\lambda(t)$ by log-likelihood maximization. Hainaut and Moraux (2018) go beyond this procedure by using their results as the initial set of parameters of a particle Markov chain Monte Carlo (PMCMC). However, in terms of log likelihood, there is no significant difference between these two procedures. We choose the POT procedure because it is less time consuming than the PMCMC procedure. The following paragraphs present, in detail, each step of our estimation procedure.

Fig. 1 The observed yield curve as of April 16, 2020, and the yield curve derived from the Hull-White model where the initial forward curve follows a Nelson-Siegel model with the estimated parameters in Table 3

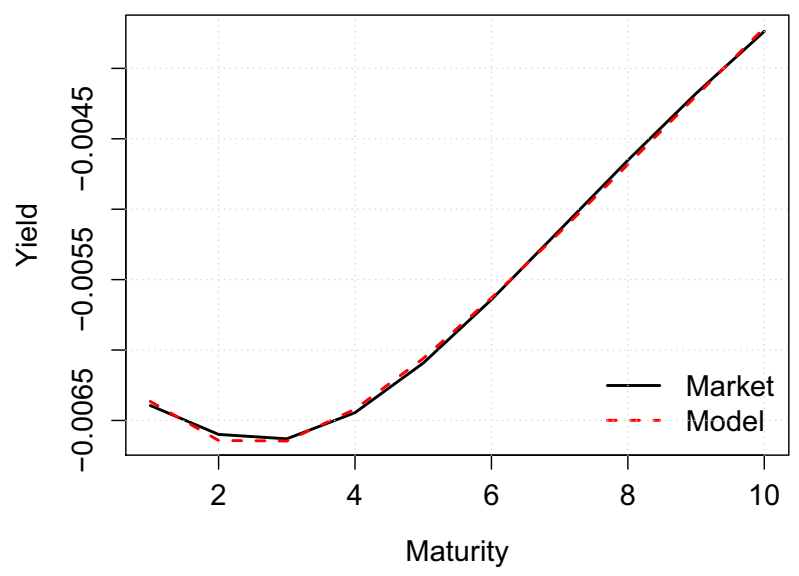


Let us denote by $y=\left\{y\left(t_{1}\right), \ldots, y\left(t_{m}\right)\right\}$ the time series of log returns of Euronext 100 , measured at times $t_{1}, \ldots, t_{m}$ and equally spaced by a lag $\Delta$ of one day of trading:

$$
y\left(t_{j}\right)=\ln \left(\frac{S\left(t_{j}\right)}{S\left(t_{j-1}\right)}\right) \quad j=1, \ldots, m .
$$

We first estimate a switching geometric Brownian motion (SGBM) process with the Hamilton filter developed by Hamilton (1989). In fact, the Kalman filter previously used for the Hull-White process has been extended by Hamilton to the case where the unobservable state variable is a Markov chain $\zeta(t)$ and where we need the probabilities that the chain will be in each state at a given time. The vector of probabilities of being at time $t_{j}$ in a certain state is computed recursively as follows:

$$
\pi_{j+1}=\frac{f\left(y\left(t_{j}\right)\right) *\left(\pi_{j}^{\top} P_{\Delta}\right)}{\left\langle f\left(y\left(t_{j}\right)\right) *\left(\pi_{j}^{\top} P_{\Delta}\right), 1_{\mathbb{R}^{N}}\right\rangle},
$$

where the initial vector $\pi_{0}$ is defined such that the chain starts with its stationary distribution which is the eigen vector of the matrix $P_{\Delta}$ associated to the eigen value equal to 1. $f\left(y\left(t_{j}\right)\right)$ is the vector of Gaussian density for $y\left(t_{j}\right)$ in each of the $N$ regimes, and $P_{\Delta}=\exp \left(Q_{0} \Delta\right)$ is the daily matrix of transition probabilities. Note that in Equation (42), * stands for the Hadamard product. Using the vectors of probabilities, the Hamilton filter's algorithm allows us to derive the following log-likelihood function for the $m$ observations of the $\log$ return:

$$
\ln L\left(y\left(t_{1}\right), \ldots, y\left(t_{m}\right)\right)=\sum_{j=1}^{m} \ln \left\langle f\left(y\left(t_{j}\right)\right),\left(\pi_{j}^{\top} P_{\Delta}\right)\right\rangle .
$$

By maximizing the log-likelihood in Equation (43), we obtain (as displayed in Table 4) the estimated parameters of the SGBM process. The SESJD process extends the SGBM process by having a modified jump part driven by the hidden Markov chain. Given the state of the chain, we expect that this jump component captures the effect of rare events on the stock prices. To detect jumps, we apply the POT procedure in the second step.

We assume that it is likely that a jump will occur when the vector $y\left(t_{j}\right)-\tilde{\mu}^{M L}\left(t_{j}\right) \Delta$ is above or below some thresholds. These thresholds are defined by:

Table 4 Parameters of a switching geometric Brownian motion, fitted with the Hamilton filter to the Euronext 100 time series

\begin{tabular}{llll}
\hline $\bar{\sigma}_{1}$ & $9.45 \%$ & $\bar{\mu}_{1}$ & $26.62 \%$ \\
$\bar{\sigma}_{2}$ & $19.89 \%$ & $\bar{\mu}_{2}$ & $-6.908 \%$ \\
$\bar{\sigma}_{3}$ & $47.78 \%$ & $\bar{\mu}_{3}$ & $-82.90 \%$ \\
$\left(p_{i j}(0,1 \text { day })\right)_{i, j=1,2,3}$ & state 1 & state 2 & state 3 \\
state 1 & 0.9587 & 0.0413 & 0.0000 \\
state 2 & 0.0074 & 0.9585 & 0.0343 \\
state 3 & 0.0000 & 0.0300 & 0.9700 \\
Log.Lik. & 12749.41 & AIC & -25474.82 \\
BIC & -25399.31 & & \\
\hline
\end{tabular}




$$
\sqrt{\Delta} \circ \Phi^{-1}\left(\alpha_{k}\right), k=1,2
$$

where $\tilde{\mu}^{M L}\left(t_{j}\right)$ is the drift of the SGBM in the most likely state obtained by the Hamilton filter, $\sigma$ is the standard deviation of the whole sample and $\Phi^{-1}\left(\alpha_{1}\right)$ and $\Phi^{-1}\left(\alpha_{2}\right)$ are respectively the $\alpha_{1}$ and $\alpha_{2}$ percentiles of the standard normal distribution. These percentiles are such that $y\left(t_{j}\right)-\tilde{\mu}^{M L}\left(t_{j}\right) \Delta$ follows a Gaussian distribution when we consider the sample without jumps or the values of log returns limited to $\Phi^{-1}\left(\alpha_{1}\right)$ and $\Phi^{-1}\left(\alpha_{2}\right)$ :

$$
\alpha_{1}, \alpha_{2}=\arg \min _{\text {sample without jumps }}\left((\text { Skewness })^{2}+(\text { Kurtosis }-3)^{2}\right) .
$$

The thresholds obtained by solving the Equation (44) are presented in Fig. 2, where the robustness of our choice is checked with the Jarque Bera test. Figure 2 enables us to link the upper or lower values of the log return to specific events, such as the credit crunch that occured between 2007 and 2009, the European sovereign debt crisis that occured between late 2009 and 2012 and the current COVID-19 crisis.

Furthermore, the POT procedure enables us to distinguish positive from negative jumps in the historical Euronext 100 prices, as in Fig. 3. Regarding our observation period, there are often negative jumps on the Euronext 100 as a result of the negative effects of crises.

Once jumps are detected, the distribution of the return on the Euronext 100 prices is approximated by $\tilde{\mu}^{M L}\left(t_{j}\right) \Delta+J_{j}$ when there is a jump at $t_{j}$. Otherwise, the return on the stock is normally distributed as

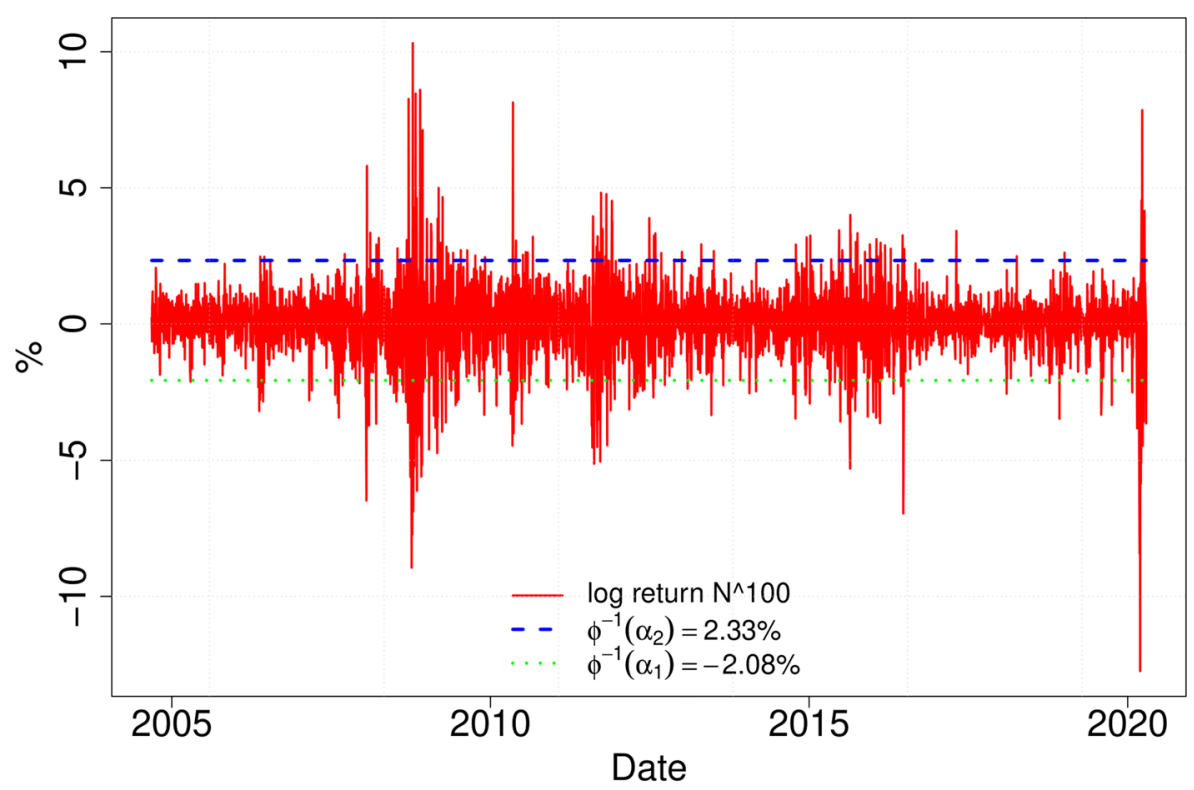

Fig. 2 The historical log return of the Euronext 100 and the thresholds between which the log return follows a Gaussian distribution. The historical log-return of the Euronext 100 prices is over the thresholds mainly during crises such as the credit crunch between 2007 and 2009, the European sovereign debt crisis between late 2009 and 2012 and currently the COVID-19 crisis 


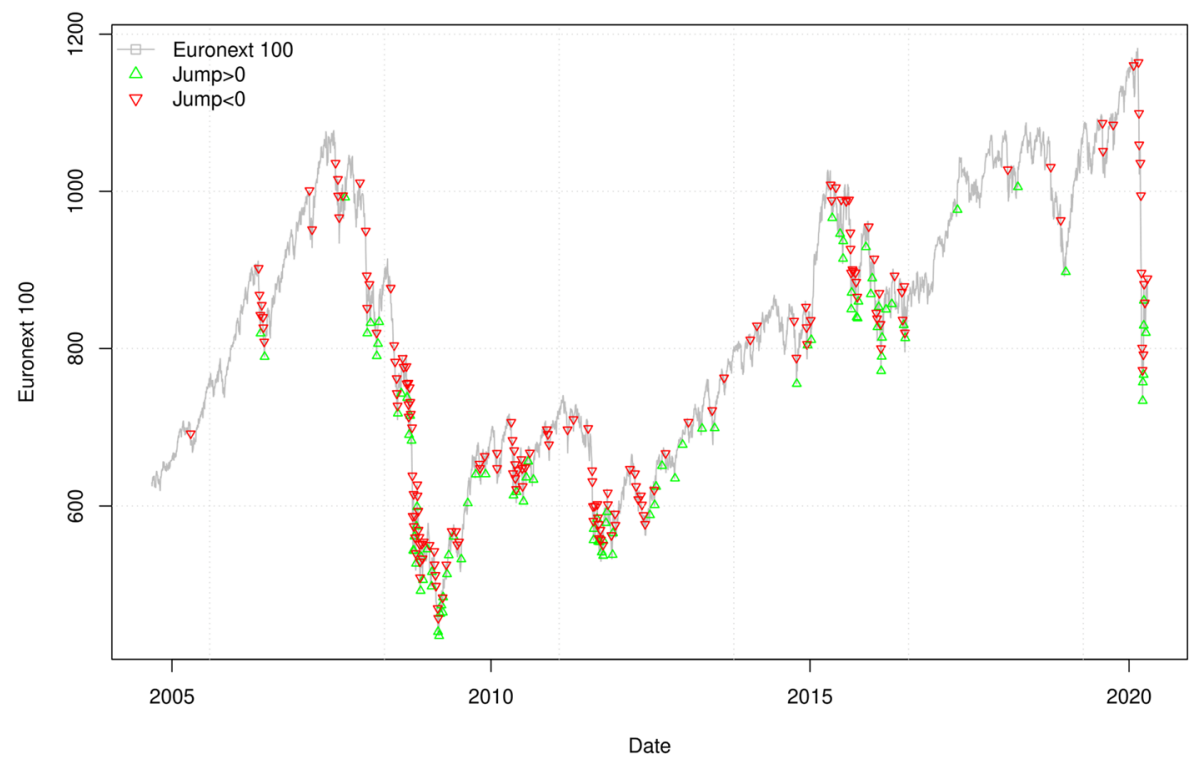

Fig. 3 The filtered jumps in the historical Euronext 100 prices. There are mainly negative jumps on the Euronext 100 prices over our observation period

$$
\tilde{\mu}^{M L}\left(t_{j}\right) \Delta+\sigma^{M L}\left(t_{j}\right)\left(\hat{\rho}+\sqrt{1-\hat{\rho}^{2}}\right) W(\Delta)
$$

where $\hat{\rho}$ is the sample correlation between the Euronext 100 prices and the Eonia rates taken as the risk-free rate proxy. $\sigma^{M L}\left(t_{j}\right)$ is the standard deviation at the most likely state obtained from the Hamilton filter procedure. We then estimate the jump parameters $\rho^{-}, \rho^{+}$ and $p$ by maximizing the log likelihood of the jump probability density function for the $\log$ return observations where jumps occur. It follows that :

$$
\rho^{-}, \rho^{+}, p=\arg \max \sum_{j=1}^{m} \ln v\left(y\left(t_{j}\right)-\tilde{\mu}^{M L}\left(t_{j}\right) \Delta \mid \rho^{-}, \rho^{+}, p\right) 1_{\left\{\text {jump at } t_{j}\right\}} .
$$

The values found are reported in Table 5. The upward exponential jumps are less likely with a probability of $36 \%$, but the average size of positive shocks $\frac{1}{\rho^{+}}$is higher than that of negative shocks $-\frac{1}{\rho^{-}}$.

Table 5 Estimated parameters of the SESJD obtained based on the log return Euronext 100 time

\begin{tabular}{llll}
\hline $\bar{\sigma}_{1}$ & $8.924 \%$ & $\bar{\mu}_{1}$ & $26.62 \%$ \\
$\bar{\sigma}_{2}$ & $16.49 \%$ & $\bar{\mu}_{2}$ & $-6.908 \%$ \\
$\bar{\sigma}_{3}$ & $18.76 \%$ & $\bar{\mu}_{3}$ & $-82.90 \%$ \\
$\bar{\eta}_{1}$ & 2.59 & $\gamma$ & 15.12 \\
$\bar{\eta}_{2}$ & 11.03 & $\beta$ & 30.09 \\
$\bar{\eta}_{3}$ & 44.82 & $p$ & 0.36 \\
$\rho^{+}$ & 31.87 & $\rho^{-}$ & -36.07 \\
Log.Lik. & 13125.87 & AIC & -26227.73 \\
BIC & -26152.22 & & \\
\hline
\end{tabular}


In the last step, we estimate the intensity parameters $\lambda(t)$. From Equation (3), over a time interval of length $\Delta$, the variation of the intensity is given by

$$
\Delta \lambda\left(t_{j}\right)=\beta\left(\eta^{M L}\left(t_{j-1}\right)-\lambda\left(t_{j-1}\right)\right) \Delta+\gamma J_{j} 1_{\left\{\text {jump at } t_{j}\right\}} \cdot
$$

We then find the estimated parameters of the intensity in Table 5 by log likelihood maximization as follows:

$$
\beta, \gamma, \bar{\eta}=\arg \max \sum_{j=1}^{m} \ln v\left(y\left(t_{j}\right) \mid \rho^{-}, \rho^{+}, p\right) 1_{\left\{\text {jump at } t_{j}\right\}} .
$$

In Figure 4, the upper graph depicts the average reversion level of the intensity and the filtered intensity obtained based on the historical Euronext 100 prices. We observe high jump intensities during crisis periods such as the credit crunch of 2008, the European sovereign debt crisis that occured between late 2009 and 2012 and the current COVID-19 crisis. During these periods, there is a large spread between jump intensities and the average reversion levels. As expected, during periods of economic growth this spread is close to zero, as is the jump intensity. Another feature is the clustering of peaks due to the self-excitation property. In the bottom graph in Figure 4, we draw the filtered state in the case of the SGBM process and the SESJD process. Their filtered state paths seem to be the same, but, during the credit crunch of 2008, the SGBM filter later detects the crisis regime, and this crisis regime lasts for a shorter period than with the SESJD filter.
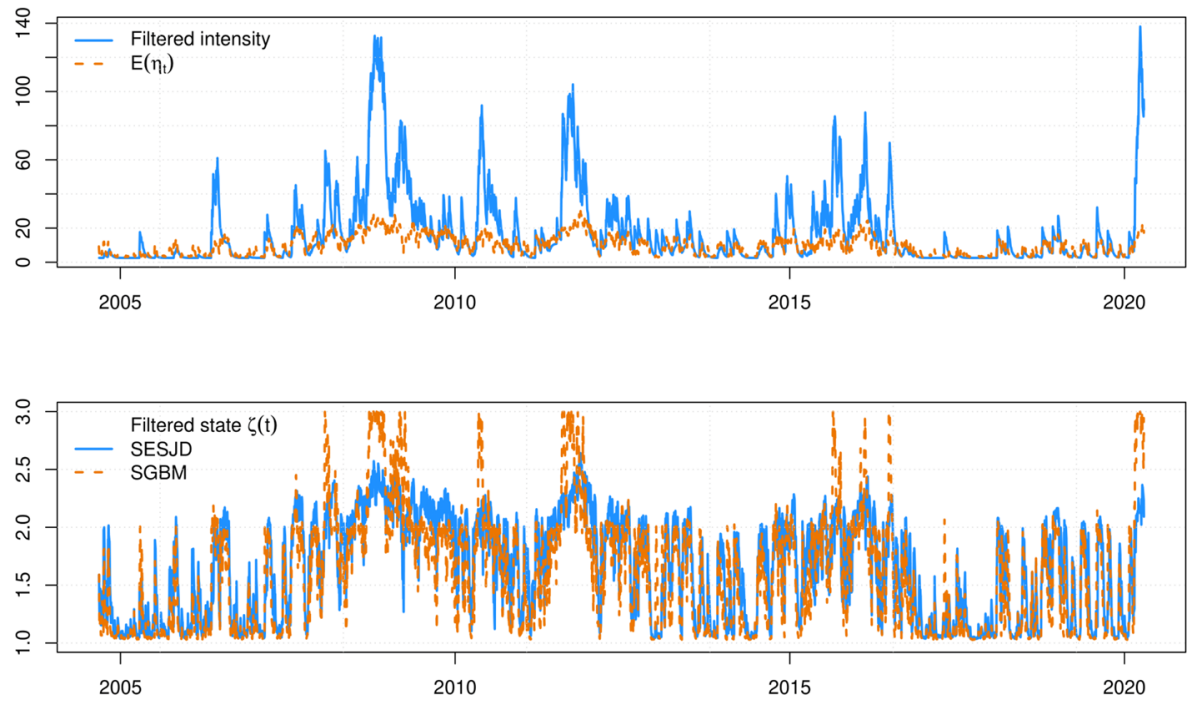

Fig. 4 The upper graph depicts the filtered intensities of the SESJD process, while the lower graph depicts the filtered states of both the SESJD and SGBM processes 
Table 6 Specification of variable annuity contract

\begin{tabular}{llll}
\hline Insured age $(x)$ & 40 years & guaranteed rate $(\delta)$ & $0 \%$ \\
\hline Premium $(\Pi)$ & 1 & Maturity $(T)$ & $\{1,2, \ldots, 20\}$ \\
\hline
\end{tabular}

\subsection{Numerical Analysis}

In this subsection, we price the value of a VA specified in Table 6 and study the impact of our setting on this price. The choice of a guaranteed rate $\delta$ equal to zero is motivated by the low interest rate level with a spot rate $r(0)=-0.65 \%$ as of April 16, 2020. The following analysis considers an insured 40-year-old person, who paid a unique premium of $€ 1$ for a GMAB and GMDB contract. To evaluate this contract, we consider the initial guaranteed amount $G(0)$ equal to the premium and we find the fair fee rate $\varphi$ solution of the Equation (26).

Figure 5 presents the fair fee rate $\varphi$ for different contract maturities. From the top left graph, the fair fee rate is not a strict monotonous function of the investment proportion in stock $\omega_{S}$, while the fair fee rate is a decreasing function of the contract maturity. The
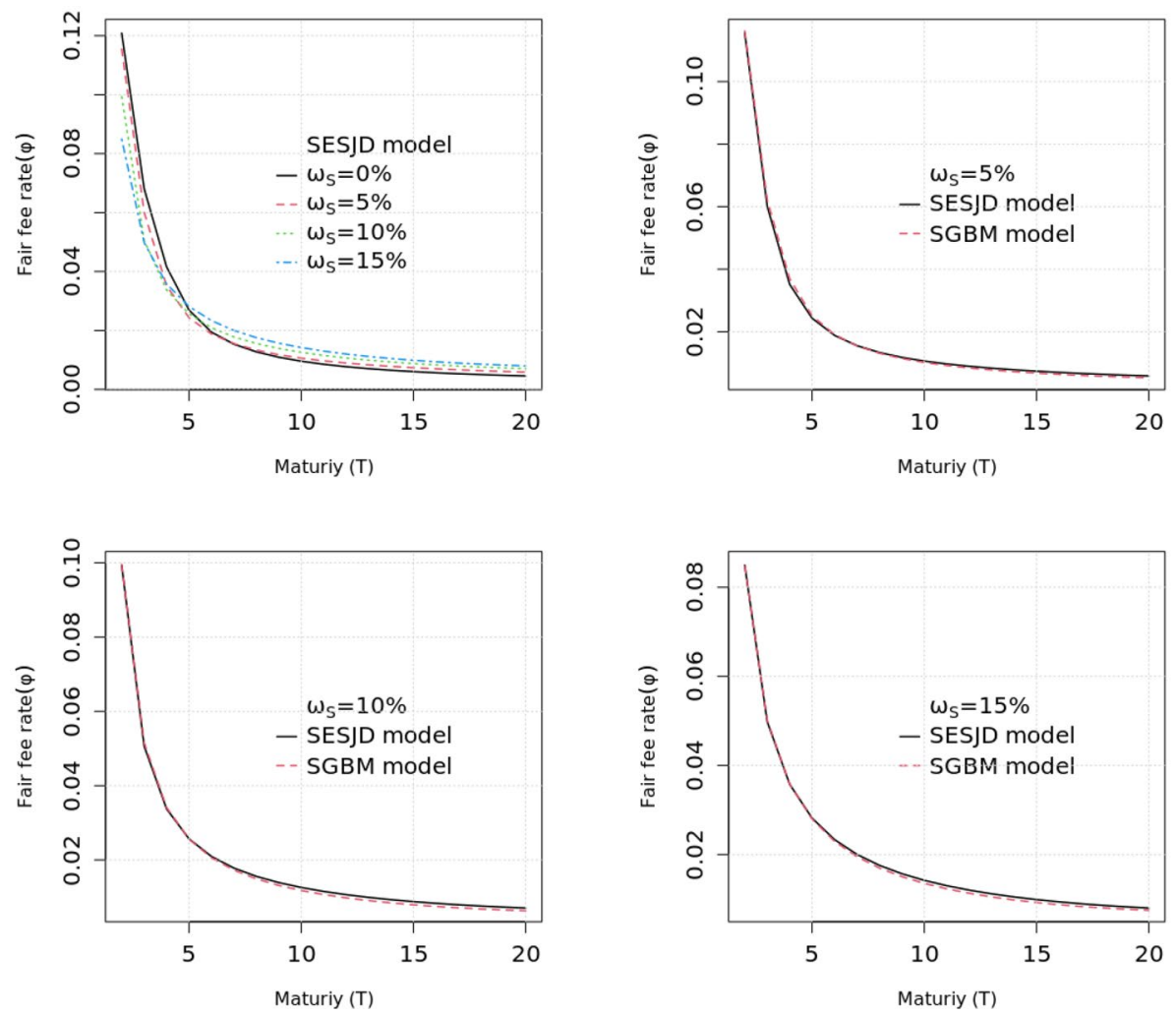

Fig. 5 Fair fee rate $\varphi$ of the GMAB and GMDB contract when we consider the roll-up guaranteed base. The guaranteed rate $\delta$ is assumed equal to zero, while the unique premium $A(0)$ is assumed equal to the initial guaranteed amount $G(0)$. The other parameters used are in Tables 4 and 5. Given the financial environment as of April 16, 2020, long term contract and a partial investment in risky asset allow to reduce the fair fee rate. For long-term contract, the SESJD model leads to a fair fee rate higher than the one from SGBM model 
GMAB and GMDB contract is then interesting for long term contract and a small investment in the Euronext 100. This is explained by positive yields on long term bonds and the cost of investment in risky assets. The top right graph and the bottom graphs compare the fair fee rates obtained using the SESJD model to those from the SGBM model for three investment scenarios, where the proportions invested in the Euronext 100 are $\omega_{S}=5 \%$, $10 \%$ and $15 \%$, respectively. For higher maturities, these graphs show that the SESJD model leads to more fees than the SGBM model. This is due to the jump component, which allows one to modulate the Euronext 100 volatility according to the economic regime. To analyze the effect of the jump component, the upper graph of Fig. 6 depicts the fair fee rates when

SESJD model, $\delta=0 \%, \omega_{\mathrm{S}}=10 \%$
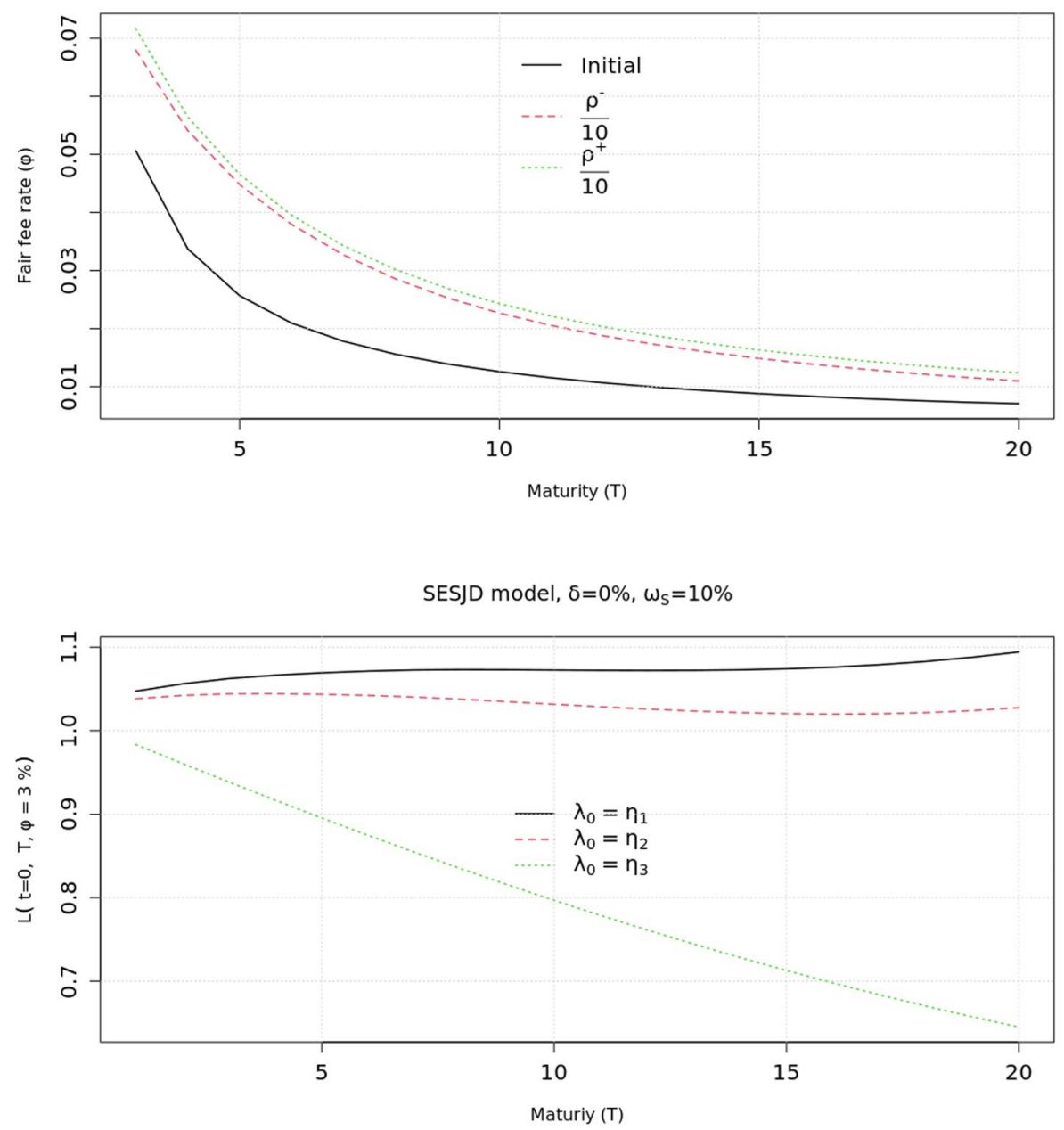

Fig. 6 Impact of the self-excitation parameters on the fair fee rate and the liability of a GMAB and GMDB contract. From the top graph, the fair fee rate is shifted up when the exponential jump parameters $\rho^{-}$and $\rho^{+}$ decrease and create more self-excitation, whereas the bottom graph shows that the liability is shifted down when the initial arrival rate of shocks $\lambda(0)=\zeta(0)^{\top} \bar{\eta}$ increases as we consider the initial regime $\zeta(0)=e_{1}$ to $e_{3}$ 
Table 7 Liability of the GMAB and GMDB contract when we consider the annual ratchet guaranteed. For each maturity $T$, we consider the fair fee rate $\varphi$ found in roll-up guaranteed base. The liability in the roll-up guaranteed base is then equal to unique premium $\Pi=1$. The liability in the annual ratchet guaranteed base obtained by Monte Carlo is higher than in roll-up guaranteed base due to the cost of additional options

\begin{tabular}{lllll}
\hline$T$ & 5 & 10 & 15 & 20 \\
\hline$\varphi(\%)$ & 2.57 & 1.26 & 0.88 & 0.71 \\
$L(t, T, \varphi)$ & 1.05 & 1.10 & 1.16 & 1.22 \\
\hline
\end{tabular}

we invest $10 \%$ in the Euronext 100 , let other parameters go unchanged and successively vary the double exponential jump parameters $\rho^{-}$and $\rho^{+}$. Recall that expectations of positive and negatives jumps are respectively equal to $\frac{1}{\rho^{-}}$and $\frac{1}{\rho^{+}}$. Divide, respectively, $\rho^{-}$and $\rho^{+}$ by 10 to increase the probability of observing large downward and upward exponential jumps, which cause more self-excitation. From the upper graph in Fig. 6, we conclude that the fair fee rate $\varphi$ increases with the level of self-excitation. The lower graph depicts, for a $3 \%$ fee rate, the liability when the initial arrival rate of shocks $\lambda(0)=\zeta(0)^{\top} \bar{\eta}$ changes given the regime $\zeta(0)=e_{1}$ to $e_{3}$. In regime 3 , which corresponds to a depressed economic context, the liability decreases dramatically with maturity due to an initial arrival rate of shocks higher than in regimes 1 and 2.

Figure 5 also reveals the difficulties of offering higher guarantees in a negative interest rate environment. From this figure, the fair fee rates range from $12 \%$ to $0.7 \%$ when we consider a guaranteed rate $\delta$ equal to zero and an initial guaranteed amount $G(0)$ equal to the unique premium. To reduce the fee rate and make the contract attractive despite low interest rates, a review of the guaranteed amount is considered. Figure 7 shows that the fair fee rate $\varphi$ decreases as $G(0)$ goes down. The negative yields in the market constrain to charge more as the guaranteed amount is higher. For short term contracts, the reduction of the initial guaranteed amount significantly reduces the fair fee rate $\varphi$ due to the uncertainty horizon.

Fig. 7 Impact of the initial guaranteed amount $G(0)$ on the fee rate $\varphi$ for the GMAB and GMDB contract. We consider the roll-up guaranteed base with a guaranteed rate $\delta=0 \%$ and a proportion invested in the Euronext 100 $\omega_{S}=10 \%$; the other parameters used are presented in Tables 4 and 5. Given the market condition as of April 16, 2020, where the spot rate $r(0)=-0.65 \%$, reducing the initial guaranteed amount allows one to reduce the fair fee rate $\varphi$

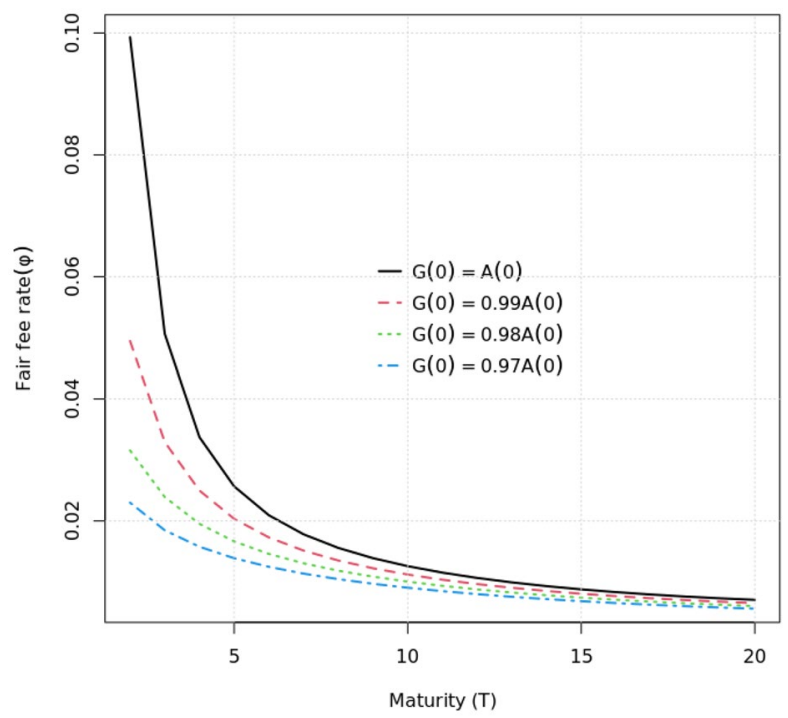




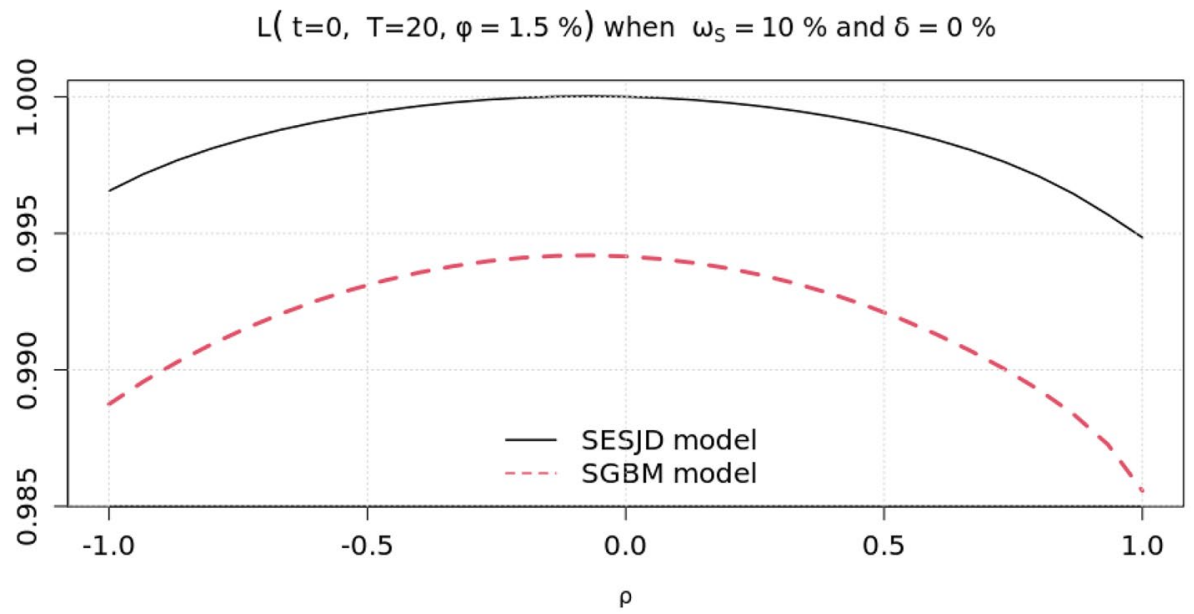

Fig. 8 Influence of the correlation between Eonia rates and Euronext 100 prices on the liability of a 20 years GMAB and GMDB contract with a $1.5 \%$ fee rate, with $10 \%$ of the premium being invested in the Euronext 100. Based on our data, the value of the liability decreases as Eonia rates and Euronext 100 prices are correlated

For the sake of comparison between roll-up guaranteed and the annual ratchet guaranteed, we compute, using the Monte Carlo approach, the liability of the GMAB and GMDB contract for a maturity $T=5,10,15,20$ and the corresponding fair fee rate found in the roll-up guaranteed. The results presented in Table 7 show that the liability in the annual ratchet guaranteed base is higher than in the roll-up guaranteed base due to the cost of additional options.

We conclude this numerical analysis with a study of the impact of the correlation between the stock market and the yield curve. We assume a 40-year-old insured person underwriting a 20-year GMAB and GMDB contract with a $1.5 \%$ fee rate and consider that $10 \%$ of the contract premium is invested in the Euronext 100. Figure 8 shows the impact of the correlation between interest rates and stock prices. In a context of low interest rates, this correlation has a small impact on liabilities, even if, from Equation (6), the market price of risk increases with the correlation $\rho$. In contrast, we observe that the value of liabilities decreases as Eonia rates and Euronext 100 prices are correlated.

\section{Conclusions}

In this article, we combined regime-switching and self-excitation features to model the underlying investment fund price of VA contracts with a minimum death and accumulated life benefit. This underlying investment fund is a portfolio of a bond and a risky asset correlated to the interest rate. Despite the dependency on the chain and the correlation between the stock and the interest rate process, we rewrote the commitment of such a VA issuer with a roll-up guaranteed base as a linear combination of put option prices. For other guaranteed bases, the dependency on the chain requires the use of the Monte Carlo approach.

This article presented a detailed economic calibration of our models based on the historical data of the Eonia rates and the Euronext 100 prices from September 06, 2004, to April 
16, 2020. The estimated parameters found for the SGBM and the SESJD processes enable us to draw three conclusions. First, ignoring the self-excitation modulated by the Markov chain leads to an underestimation of the fair fee rate of the VA contract. The features of the SESJD process are thus recommended, as they allow one to not only consider the economic regime, but also to capture random shocks in the stock market and to scale their amplitude given the regime. Second, in a low interest rate environment, reducing the guarantee allows one to decrease the fair fee rate. Finally, the GMAB and GMDB contracts are sensitive to the economic regime and the self-excitation level.

From a broader perspective, the article calls for consideration of other guarantees such as the surrender benefit or the withdrawal benefit. From a practical perspective, it would be interesting to extend this work to the valuation of a large portfolio of VA contracts based on risk measures.

\section{Appendix}

\section{Appendix A}

The probability function (pdf) $v(z)$ is defined by the three parameters $\rho^{+} \in \mathbb{R}^{+}, \rho^{-} \in \mathbb{R}^{-}$and $p \in(0,1)$ :

$$
v(z)=p \rho^{+} e^{-\rho^{+} z} 1_{\{z \geq 0\}}-(1-p) \rho^{-} e^{-\rho^{-} z} 1_{\{z<0\}},
$$

where $p$ and $(1-p)$ are respectively the probabilities of observing upward and downward exponential jumps.

The average sizes of positive and negative shocks are equal to $\frac{1}{\rho^{+}}$and $\frac{1}{\rho^{-}}$. The expectation of $J_{i}$ is the weighted sum of the expected average jumps:

$$
\mathbb{E}\left(J_{i}\right)=p \frac{1}{\rho^{+}}+(1-p) \frac{1}{\rho^{-}} .
$$

In later developments, the moment-generating function for the sum of the $J_{i}$ and of its absolute value is needed. We define it as follows:

$$
\begin{aligned}
\psi_{J}\left(z_{1}, z_{2}\right) & :=\mathbb{E}\left(e^{z_{1} J+z_{2}|J|}\right) \\
& =p \frac{\rho^{+}}{\rho^{+}-\left(z_{1}+z_{2}\right)}+(1-p) \frac{\rho^{-}}{\rho^{-}-\left(z_{1}-z_{2}\right)},
\end{aligned}
$$

under the condition that $\left(z_{1}+z_{2}\right)<\rho^{+}$and $\left(z_{1}+z_{2}\right)>\rho^{-}$. In particular,

$$
\mathbb{E}\left(e^{J}-1\right)=p \frac{\rho^{+}}{\rho^{+}-1}+(1-p) \frac{\rho^{-}}{\rho^{-}-1}-1 \text {. }
$$

\section{Appendix B}

If we note $f(t, s, r(t), \lambda(t), X(t), \zeta(t))=\mathbb{E}^{\mathbb{Q}}\left(e^{\omega_{1} \int_{t}^{s} r(u) \mathrm{d} u+\omega_{2} X(s)} \mid \mathcal{F}_{t}\right), f$ is the solution for an Ito's equation for the semi-martingale. Let $\mathcal{A}$ the infinitesimal generator of $(r(t), \lambda(t), X(t), \zeta(t))$. By definition, this infinitesimal generator equals 


$$
\lim _{u \rightarrow t} \frac{\mathbb{E}^{\mathbb{Q}}\left(f(u, s, r(u), \lambda(u), X(u), \zeta(u)) \mid \mathcal{F}_{t}\right)-f(t, s, r(t), \lambda(t), X(t), \zeta(t))}{u-t} .
$$

With enough regularity we have:

$$
\mathcal{A} f(t, s, r(t), \lambda(t), X(t), \zeta(t))=-\omega_{1} r(t) f(t, s, r(t), \lambda(t), X(t), \zeta(t)) .
$$

Since $f(t, s, r(t), \lambda(t), X(t), \zeta(t))$ is the solution of an Ito's equation for the semimartingale, if $\zeta(t)=e_{i}, i \in \mathcal{N}$, then:

$$
\begin{aligned}
-\omega_{1} r(t) f & =f_{X}\left(r(t)-\omega_{S} \lambda(t) \mathbb{E}^{\mathbb{Q}}\left(e^{J}-1\right)\right. \\
& \left.-\frac{1}{2}\left(\left(\omega_{S} \rho \bar{\sigma}_{i}-\left(1-\omega_{S}\right) \sigma_{r} B^{r}(t, T)\right)^{2}+\omega_{S}^{2}\left(1-\rho^{2}\right) \bar{\sigma}_{i}^{2}\right)\right)+f_{t} \\
& +\left(\theta^{\mathbb{Q}}(t)-a_{r} r(t)\right) f_{r}+\frac{1}{2} \sigma_{r}^{2} f_{r r}-\sigma_{r}\left(1-\omega_{S}\right) \sigma_{r} B^{r}(t, T) f_{r X} \\
& +\sigma_{r} \omega_{S} \rho \bar{\sigma}_{i} f_{r X}+\frac{1}{2}\left(\left(\omega_{S} \rho \bar{\sigma}_{i}-\left(1-\omega_{S}\right) \sigma_{r} B^{r}(t, T)\right)^{2}\right) f_{X X} \\
& +\frac{1}{2} \omega_{S}^{2}\left(1-\rho^{2}\right) \bar{\sigma}_{i}^{2} f_{X X}+\beta\left(\bar{\eta}_{i}-\lambda(t)\right) f_{\lambda}+\lambda(t) \\
& \times \int_{-\infty}^{+\infty}\left(f\left(t, s, r(t), \lambda(t)+\gamma|z|, X(t)+\ln \left(1+\omega_{S}\left(e^{z}-1\right)\right), e_{i}\right)-f(\cdot)\right) v(\mathrm{~d} z) \\
& +\sum_{j \neq i}^{\mathcal{N}} q_{i, j}\left(f\left(t, s, r(t), \lambda(t), X(t), e_{j}\right)-f\left(t, s, r(t), \lambda(t), X(t), e_{i}\right)\right) .
\end{aligned}
$$

${ }^{4}$ Assume that $f$ is an exponential affine function of $r(t), \lambda(t)$ and $X(t)$ :

$$
\begin{aligned}
f\left(t, s, r(t), \lambda(t), X(t), e_{i}\right)= & \exp \left(A\left(\bar{\omega}, t, s, e_{i}\right)+B(\bar{\omega}, t, s) r(t)\right. \\
& \left.+C(\bar{\omega}, t, s) \lambda(t)+\omega_{2} X(t)\right),
\end{aligned}
$$

where $\bar{\omega}=\left(\omega_{1}, \omega_{2}\right) . A\left(\bar{\omega}, t, s, e_{i}\right)$ for $i \in \mathcal{N}, B(\bar{\omega}, t, s)$ and $C(\bar{\omega}, t, s)$ are time dependent functions. Since $f(s, s, r(s), \lambda(s), X(s), \zeta(s))=e^{\omega_{2} X(s)}$ we have the following terminal condition $A\left(\bar{\omega}, s, s, e_{i}\right)=0$ for $i \in \mathcal{N}, B(\bar{\omega}, s, s)=0$ and $C(\bar{\omega}, s, s)=0$. We can simplify the two last terms of Equation (46) as follows:

$$
\begin{array}{r}
\int_{-\infty}^{+\infty}\left(f\left(t, s, r(t), \lambda(t)+\gamma|z|, X(t)+\ln \left(1+\omega_{S}\left(e^{z}-1\right)\right), e_{i}\right)-f(\cdot)\right) v(\mathrm{~d} z)= \\
f\left(t, s, r(t), \lambda(t), X(t), e_{i}\right)\left(\mathbb{E}^{\mathbb{Q}}(g(J, C))-1\right)
\end{array}
$$

where $g:(x, C) \longmapsto e^{\omega_{2} \ln \left(1+\omega_{S}\left(e^{x}-1\right)\right)+C \gamma|x|}$ is a real valued bivariate function. As shown in Appendix $C$, this function admits a closed-form expression when $\omega_{2}=1$. Since $q_{i i}=-\sum_{j \neq i, j \in \mathcal{N}} q_{i, j}$, the last term becomes:

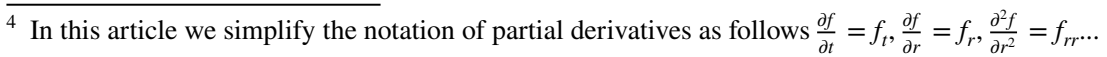




$$
\begin{array}{r}
\sum_{j \neq i, j \in \mathcal{N}} q_{i, j}\left(f\left(t, s, r(t), \lambda(t), X(t), e_{j}\right)-f\left(t, s, r(t), \lambda(t), X(t), e_{i}\right)\right)= \\
\sum_{j \in \mathcal{N}} q_{i, j} f\left(t, s, r(t), \lambda(t), X(t), e_{j}\right) .
\end{array}
$$

Then, from Equation (46), we derive this PDEs system:

$$
\left\{\begin{aligned}
0= & A_{t}+\theta^{\mathbb{Q}}(t) B+\frac{1}{2} \sigma_{r}^{2} B^{2}+\sigma_{r}\left(\omega_{S} \rho \bar{\sigma}_{i}-\left(1-\omega_{S}\right) \sigma_{r} B^{r}(t, T)\right) \omega_{2} B \\
& \quad-\frac{\omega_{2}}{2}\left(\left(\omega_{S} \rho \bar{\sigma}_{i}-\left(1-\omega_{S}\right) \sigma_{r} B^{r}(t, T)\right)^{2}+\omega_{S}^{2}\left(1-\rho^{2}\right) \bar{\sigma}_{i}^{2}\right) \\
+ & \frac{\omega_{2}^{2}}{2}\left(\left(\omega_{S} \rho \bar{\sigma}_{i}-\left(1-\omega_{S}\right) \sigma_{r} B^{r}(t, T)\right)^{2}+\omega_{S}^{2}\left(1-\rho^{2}\right) \bar{\sigma}_{i}^{2}\right)+\beta \bar{\eta}_{i} C \\
+ & \sum_{j \in \mathcal{N}} q_{i, j} \exp \left(A\left(\bar{\omega}, t, s, e_{j}\right)-A\left(\bar{\omega}, t, s, e_{i}\right)\right) \quad \text { for } \quad i \in \mathcal{N} \\
0= & B_{t}-a_{r} B+\omega_{2}+\omega_{1} \\
0= & C_{t}-\omega_{2} \omega_{S} \mathbb{E}^{\mathbb{Q}}\left(e^{J}-1\right)-\beta C+\mathbb{E}^{\mathbb{Q}}(g(J, C))-1
\end{aligned}\right.
$$

Let $\tilde{A}(\bar{\omega}, t, s)=\left(e^{A\left(\bar{\omega}, t, s, e_{1}\right)}, \ldots, e^{A\left(\bar{\omega}, t, s, e_{N}\right)}\right)$ be a vector of functions. Then, the first equation of the System (48) has the following matrix form:

$$
\frac{\partial \tilde{A}(\bar{\omega}, t, s)}{\partial t}+\left(\operatorname{diag}(D(\bar{\omega}, t, s))+Q_{0}\right) \tilde{A}(\bar{\omega}, t, s)=0
$$

where $D(\bar{\omega}, t, s)$ is a vector of functions defined by

$$
\begin{aligned}
D(\bar{\omega}, t, s)=- & \frac{\omega_{2}}{2}\left(\left(\omega_{S} \rho \bar{\sigma}-\left(1-\omega_{S}\right) \sigma_{r} B^{r}(t, T)\right)^{2}+\omega_{S}^{2}\left(1-\rho^{2}\right) \bar{\sigma}^{2}\right) \\
+ & \theta^{\mathbb{Q}}(t) B+\frac{1}{2} \sigma_{r}^{2} B^{2}+\sigma_{r}\left(\omega_{S} \rho \bar{\sigma}-\left(1-\omega_{S}\right) \sigma_{r} B^{r}(t, T)\right) \omega_{2} B \\
& +\frac{\omega_{2}^{2}}{2}\left(\left(\omega_{S} \rho \bar{\sigma}-\left(1-\omega_{S}\right) \sigma_{r} B^{r}(t, T)\right)^{2}+\omega_{S}^{2}\left(1-\rho^{2}\right) \bar{\sigma}^{2}\right)+\beta \bar{\eta} C .
\end{aligned}
$$

We complete the proof of this proposition with the solution of the second equation of the PDEs system (48):

$$
B(\bar{\omega}, t, s)=\left(\omega_{2}+\omega_{1}\right) B^{r}(t, s) .
$$

\section{Appendix C}

For a real-valued function $C(\cdot)$ and $\omega_{2}=1$, if $g:(x, C) \longmapsto e^{\omega_{2} \ln \left(1+\omega_{S}\left(e^{x}-1\right)\right)+C \gamma|x|}$

$$
\begin{aligned}
\mathbb{E}^{\mathbb{Q}}(g(J, C)) & =\mathbb{E}^{\mathbb{Q}}\left(e^{\ln \left(1+\omega_{S}\left(e^{J}-1\right)\right)+C(\bar{\omega}, t, s) \gamma|J|}\right) \\
& =\mathbb{E}^{\mathbb{Q}}\left(\left(1+\omega_{S}\left(e^{J}-1\right)\right) e^{C(\bar{\omega}, t, s) \gamma|J|}\right) \\
& =\left(1-\omega_{S}\right) \psi_{J}(0, \gamma C(\bar{\omega}, t, s))+\omega_{S} \psi_{J}(1, \gamma C(\bar{\omega}, t, s)) .
\end{aligned}
$$




\section{Appendix D}

Using Simpson's rule, we approximate the integral in Equation (20) as follows :

$$
p_{T}(k) \approx \frac{e^{\alpha k}}{\pi} \sum_{l=1}^{M} \delta_{l} e^{-i v_{l} k} \mathcal{F}\left(\bar{p}_{T}\right)\left(v_{l}\right) \Delta_{v}
$$

where $\delta_{l}=\frac{1}{3}\left(3+(-1)^{j}-1_{\{j=1\}}\right)$. The Fourier transform of the modified put option $\mathcal{F}\left(\bar{p}_{T}\right)\left(v_{l}\right)$ is determined as follows:

$$
\begin{aligned}
\frac{\mathcal{F}\left(\bar{p}_{T}\right)\left(v_{l}\right)}{V(t)} & =\int_{-\infty}^{+\infty} e^{i v_{l} k} \int_{-\infty}^{k} \int_{-\infty}^{+\infty} e^{-y_{1}}\left(e^{(1-\alpha) k}-e^{y_{2}-\alpha k}\right) \psi\left(y_{1}, y_{2}\right) \mathrm{d} y_{1} \mathrm{~d} y_{2} \mathrm{~d} k \\
& =\int_{-\infty}^{+\infty} \int_{-\infty}^{+\infty} \int_{y_{2}}^{+\infty} e^{i v_{l} k-y_{1}}\left(e^{(1-\alpha) k}-e^{y_{2}-\alpha k}\right) \psi\left(y_{1}, y_{2}\right) \mathrm{d} k \mathrm{~d} y_{1} \mathrm{~d} y_{2} \\
& =\int_{-\infty}^{+\infty} \int_{-\infty}^{+\infty} e^{-y_{1}} \psi\left(y_{1}, y_{2}\right)\left(\frac{e^{\left(1-\alpha+i v_{l}\right) y_{2}}}{i v_{l}-\alpha}-\frac{e^{\left(1-\alpha+i v_{l}\right) y_{2}}}{1+i v_{l}-\alpha}\right) \mathrm{d} y_{1} \mathrm{~d} y_{2} \\
& =\frac{M_{r, X}^{t, T}\left(-1,1-\alpha+i v_{l}\right)}{\alpha^{2}-\alpha-v_{l}^{2}+i(-2 \alpha+1) v_{l}}
\end{aligned}
$$

From Equation (19), $\mathcal{F}\left(\bar{p}_{T}\right)(0)$ should be finite, and $\alpha$ is non-negative. It follows that the put option price is well-defined for any $\alpha>0$ such that $M_{r X}^{t, T}(-1,1-\alpha)<+\infty$.

In contrast, for $k_{j}=-k_{\max }+(j-1) \Delta_{k}, \Delta_{k}=\frac{2 k_{\max }}{M}, \Delta_{v}=\frac{r_{2 \pi}}{M \Delta_{k}}$ and $v_{l}=(l-1) \Delta_{v}$, the product $v_{l} k_{j}$ is equal to

$$
\begin{aligned}
v_{l} k_{j} & =-\frac{M}{2}(l-1) \Delta_{v} \Delta_{k}+(j-1)(l-1) \Delta_{v} \Delta_{k} \\
& =-(l-1) \pi+(j-1)(l-1) \frac{2 \pi}{M},
\end{aligned}
$$

and

$$
e^{-i v_{l} k}=(-1)^{l-1} e^{-\frac{2 \pi i}{M}(j-1)(l-1)}
$$

Equations (50) and (52) to (49) allow us to end this proof.

\section{References}

Anna RB, Pietro M, Annamaria O, Ermanno P (2011) Variable annuities: A unifying valuation approach. Insurance Math Econom 49:285-297

Babbs S , Nowman K (1999) Kalman filtering of generalized vasicek term structure models, J Financial Quantitative Analysis. 34

Barbarin J, Devolder P (2005) Risk measure and fair valuation of an investment guarantee in life insurance. Insurance Math Econom 37:297-323

Bauer D, Kling A, Russ J (2008) A universal pricing framework for guaranteed minimum benefits in variable annuities. ASTIN Bulletin 38(2):621-651

Bernard C, Hardy M, Mackay A (2014) State-dependent fees for variable annuity guarantees. ASTIN Bulletin 44(3):559-585

Bernard C, Moenig T (2019) Where Less Is More: Reducing Variable Annuity Fees to Benefit Policyholder and Insurer. Journal of Risk and Insurance 86(3):761-782 
Boyle PP, Schwartz ES (1977) Equilibrium prices of guarantees under equity-linked contracts. J Risk Insur 44(4):639

Brennan MJ, Schwartz ES (1976) The pricing of equity-linked life insurance policies with an asset value guarantee. J Financ Econ 3(3): 195-213

Brennan MJ, Schwartz ES (1979) Alternative investment stragtegies for the issuers of equity-linked life insurance with an asset value guarantee. J Bus 52:63-93

Cox JC, Ingersoll JE, Ross S (1985) A Theory of the Term Structure of Interest Rates. Econometrica 53(2):385-407

Cui Z, Feng R, MacKay A (2017) Variable Annuities with VIX-Linked Fee Structure under a Heston-Type Stochastic Volatility Model. North American Actuarial J 21(3):458-483

David L, Bin L, Yumin W (2021) High-water mark fee structure in variable annuities. J Risk Insurance, 1-38. https://doi.org/10.1111/jori.12345

Elliott RJ, Chan L, Siu TK (2005) Option pricing and esscher transformation under regime regime switching. Ann Finance 1(4):423-432

Goldfeld SM, Quandt RE (1973) A markov model for switching regressions. J Econ 1(1):3-15

Gompertz B (1825) On the Nature of the Function Expressive of the Law of Human Mortality, and on a New Mode of Determining the Value of Life Contingencies. Philos Trans R Soc Lond 115:513-583

Hainaut D (2014) Impulse control of pension fund contributions, in a regime switching economy. Eur J Oper Res 239:810-819

Hainaut D, Colwell D (2016) A structural model for credit risk with markov modulated lévy processes and synchronous jumps. Eur J Financ 22(11):1040-1062

Hainaut D, Moraux F (2018) A switching self-exciting jump diffusion process for stock prices. Ann Finance 15(2):267-306

Hamilton JD (1989) A new approach to economic analysis of non-stationnary time series and the business cycle. Econometrica 57:357-384

Hardy MR (2001) A regime-switching model of long-term stock returns. North American Actuarial J $5(2): 41-53$

Hardy M (2003) Investment Guarantees: Modeling and Risk Management for Equity-Linked. Life Insurance, Wiley Finance

Hull J, White A (1990) Pricing Interest-Rate-Derivative Securities. Rev Finan Stud 3(4):573-592

Ignatieva K, Andrew S, Ziveyi J (2016) Pricing and hedging of guaranteed minimum benefits under regimeswitching and stochastic mortality. Insurance Math Econom 70:286-300

Kalman R (1960) A New Approach to Linear Filtering and Prediction Problems. ASME J Basic Eng $82: 35-45$

Kijima M (2007) Wong T (2007) Pricing of ratchet equity-indexed annuities under stochastic interest rates. Insurance Math Econom 41(3):317-338

Kun F, Yang S, Tak KS, Rongming W (2015) Pricing annuity guarantees under a double regime-switching model. Insurance Math Econom 62:62-78

Makeham WM (1860) One the Law of Mortality and the Construction of Annuity Tables. The Assurance Magazine, and J Inst Actuaries 8(6):301-310

Milvesky M, Posner SE (2001) The titanic option: Valuation of the guaranteed minimum death benefit in variable annuities and mutual funds. J Risk Insurance 68(1):91-126

Milvesky M, Salisbury TS (2006) Financial valuation of the guaranteed minimum withdrawal benefits. Insurance Math Econom 38:21-38

Nelson CR, Siegel AF (1987) Parsimonious Modeling of Yield Curves. J Bus 60(4):473-489

Oldrich V (1977) An equilibrium characterization of the term structure. J Financ Econ 5(2):177-188

Runhuan F, Hans WV (2012) Analytical calculation of risk measures for variable annuity guaranteed benefits. Insurance Math Econom 51(3):636-648

Sheldon XL, Tan KS, Hailiang Y (2009) Pricing annuity guarantees under a regime-switching model. North American Actuarial J 13(3):316-332

Sheldon XL, Tan KS (2003) Valuation of equity-indexed annuities under stochastic interest rates. North American Actuarial J 7(4):72-91

Siu TK (2005) Fair valuation of participating policies with surrender options and regime regime switching. Insurance Math Econom 37(3):553-552

Publisher's Note Springer Nature remains neutral with regard to jurisdictional claims in published maps and institutional affiliations. 